Chapman University

Chapman University Digital Commons

Computational and Data Sciences (MS) Theses

Dissertations and Theses

Fall 12-4-2019

\title{
Development and Validation of Wearable Systems for Human Postural Sway Analysis
}

\author{
Michael Pollind \\ Chapman University, polli104@mail.chapman.edu
}

Follow this and additional works at: https://digitalcommons.chapman.edu/cads_theses

\section{Recommended Citation}

M. Pollind, "Development and validation of wearable systems for human postural sway analysis," M. S. thesis, Chapman University, Orange, CA, 2019. https://doi.org/10.36837/chapman.000110

This Thesis is brought to you for free and open access by the Dissertations and Theses at Chapman University Digital Commons. It has been accepted for inclusion in Computational and Data Sciences (MS) Theses by an authorized administrator of Chapman University Digital Commons. For more information, please contact laughtin@chapman.edu. 


\title{
Development and Validation of Wearable Systems for Human Postural Sway Analysis
}

\author{
A Thesis by \\ Michael L. Pollind \\ Chapman University \\ Orange, CA \\ December 2019 \\ Committee in charge: \\ Chair - Rahul Soangra \\ Mohamed Allali \\ Niklas Ignasiak \\ Jo Armour Smith
}

Schmid College of Science and Technology

Submitted in partial fulfillment of the requirements for the degree of

Master of Science in Computational \& Data Sciences 
The Thesis of Michael L. Pollind is approved

December 2019

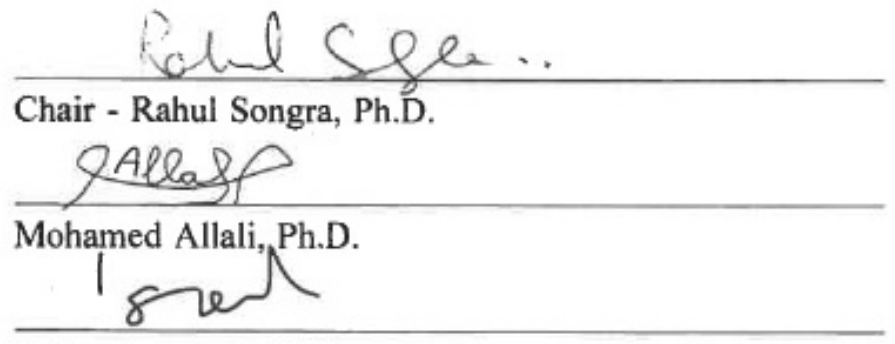

Niklas Ignasiak, Ph.D.

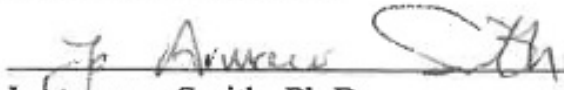

Jo Armour Smith, Ph.D. 


\title{
Development and Validation of Wearable Systems for Human Postural Sway Analysis
}

Copyright (C 2019

\author{
by Michael L. Pollind
}




\section{DEDICATION}

I would like to dedicate this work to my parents for their social and economic support for getting through university. Also the support of the professors at Chapman University and their continued help along with counseling and support. 


\section{ACKNOWLEDGEMENTS}

I would like to acknowledge, Christopher Hoang, Michael Shiraishi , Henery Do and the Gait Rehabilitation Research Laboratory for their support in helping me put this together along with support from student body from Rinker. Henery Do and Christopher Hoang help design some of the enclosures for these devices along with organizing these experimental setup with Dr. Soangra. Michael Shiraishi Helped with collecting data for the synthetic data for the RQA analysis. Their continued support helped me put together this educational work and also provided the resources to develop these tools and processes for the lab. 


\title{
ABSTRACT \\ Development and Validation of Wearable Systems for Human Postural Sway Analysis
}

\author{
by Michael L. Pollind
}

Falls are the most common cause of injury in older adults. Around one-third of senior citizens (aged 65 or over) experience at least one fall per year, and the frequency increases by 66 percent for those aged over 85 years. Nowadays wearable systems are gaining popularity to perform fall risk assessments and investigating fall events in natural environments. However all commercially existing systems are expensive, thus there is paucity of knowledge to develop and validate inexpensive wearable systems for fall risk assessment in older adults. An early risk of fall assessment could help health care professionals to intervene earlier. This study investigates the processes involved with designing an Inertial Measurement Unit (IMU) including the rational behind the choice of parts and assembly of the board. The final sensor developed (Mini-Logger) was validated for sway acquisition in laboratory setting. Further the novel sensor was tested on healthy adults for its sensitivity with postural sway at 12 different standing conditions. The results from this study could help in development of inexpensive wearable systems which could identify older individuals at risk of falling for proactive fall prevention. Thus reduction in falls will improve the quality of life of older adults and thereby reduce healthcare costs. 


\section{TABLE OF CONTENTS}

1 Introduction $\quad 1$

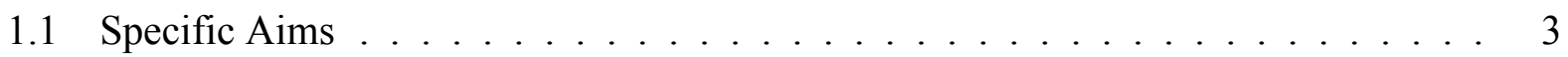

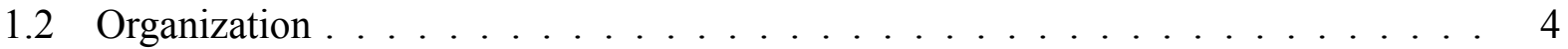

2 Hardware $\quad 5$

2.1 Mini Data Logger . . . . . . . . . . . . . . . . . . . 5

2.2 Materials and Methods . . . . . . . . . . . . . . . . . 5

2.3 Results . . . . . . . . . . . . . . . . . . . . 10

2.4 Discussion . . . . . . . . . . . . . . . . . . . 11

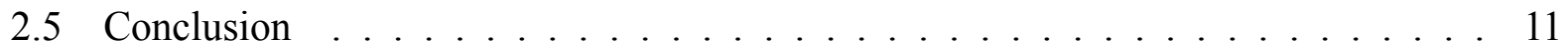

3 Postural Sway Assessment from Inertial Sensors With Human Subjects 13

3.1 Introduction . . . . . . . . . . . . . . . . . . . . 13

3.2 Materials and Methods . . . . . . . . . . . . . . . . . 15

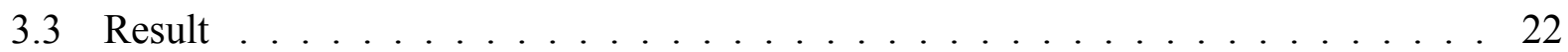

3.4 Discussion . . . . . . . . . . . . . . . . . . . 24

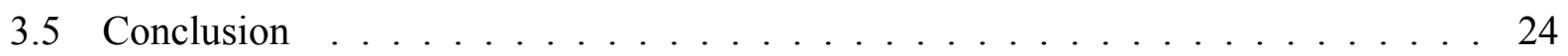

4 Validation of Sway using Motion Capture Camera System and Inertial Sensors 28

4.1 Introduction . . . . . . . . . . . . . . . . . . . . 28

4.2 Methods and Materials . . . . . . . . . . . . . . . . . . 31

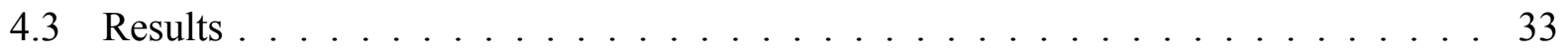

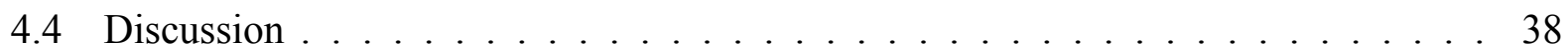

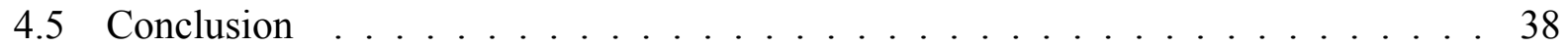

5 Conclusion $\quad 39$

$\begin{array}{ll}\text { Appendix } & 41\end{array}$

$\begin{array}{ll}\text { References } & 44\end{array}$ 


\section{LIST OF TABLES}

3.1 Different conditions of postural standing . . . . . . . . . . . . 16

3.2 Mini Logger Results from MANOVA . . . . . . . . . . . . . . . . . 25

3.3 Xsens Results from MANOVA . . . . . . . . . . . . . . . 26

4.1 result for determinism and recurrence rate . . . . . . . . . . . . 37 


\section{LIST OF FIGURES}

2.1 Mini-Logger . . . . . . . . . . . . . . . . . . . . . 6

2.2 ICM-20948 recommend schematic from datasheet (ICM-20948) . . . . . . . . 6

2.3 SD card pinout . . . . . . . . . . . . . . . . . 8

3.1 Logger Mounting . . . . . . . . . . . . . . . . . . . 16

3.2 Experimental Setup . . . . . . . . . . . . . . . . . . . 17

3.3 Filtered IMU Data . . . . . . . . . . . . . . . . . . . . 22

3.4 Comparison of mini logger and xsens for a given trial. . . . . . . . . . . . . 23

4.1 Recurrence Plot from synthetic motion . . . . . . . . . . . . . . . . . . . 29

4.2 Sway Analysis Setup . . . . . . . . . . . . . . . . . . . . 32

4.3 Treadmill Sensor Mounting . . . . . . . . . . . . . . . . . . 32

4.4 Filtered $x$ and $y$ data from Xsens and Vicon from synthetic motion . . . . . . . . 33

4.5 RQA subset from figure $4.1 \ldots \ldots \ldots \ldots$

4.6 Recurrence rate . . . . . . . . . . . . . . . . . . . 35

4.7 Determinism relation to frequency using a minimum diagonal length of 5 units. Vicon and Xsens shows similar profiles when combination of amplitude and frequency are combined, but xsens suffers more from signal noise. . . . . . . . . . . . . 35 


\section{CHAPTER 1}

\section{Introduction}

Balance is an essential skill for functional mobility which generally degrades with advanced age (Shaffer \& Harrison, 2007). Balance problems in older individuals increases the risk of fall related injuries, increasing the likely-hood morbidity and mortality (Shaffer \& Harrison, 2007). A third of individuals over 65 experience at least one fall each year and the cost is expected to reach $\$ 32$ billion by 2020 (Shaffer \& Harrison, 2007). 1 in 40 indviduals are at risk of falling every year and as a result Unintentional injury is considered the $5^{\text {th }}$ leading cause of death in older individuals after cardiovascular disease, cancer, stroke, and pulmonary disorders (Rubenstein, 2006, Zalewski, 2015). Understanding the factors that contribute to fall risk can aid these individuals. Understanding postural balance is a strong tool that clinicians can use to identify at risk individuals.

The nature of standing upright is inherently unstable and small deviations created due to postural muscles firing and gravational induced joint torques can unbalance the process (Peterka, 2002). Corrective torques are added to recenter the off centering effects of gravity and this creates a pattern of motion that is measured by Center of Pressure (COP) (Peterka, 2002).

$\mathrm{COP}$ is a strong measurement of stability and can be used as one important factor to asses fall 
risk (Liu et al., 2012). There are three sensory systems that are used to assess stability and they included: Visual, Vestibular, and Somatosensory. Force plates are the gold standard for assessing center of pressure but due to weight, size, cost and maintenance they might not be available in every setting (Liu et al., 2012, Ekvall Hansson et al., 2019). The price of a single IMU (Inertial Measurement Units) is considerably more affordable when compared with using a force plate. previous assessments have shown that an force plates and IMU's show similar characteristics (Ekvall Hansson et al., 2019).

In this study, we build a custom IMU from common off the shelf components and assembled by a third party. This works tries to understand the feasibility of developing and verifying a custom built sensor when compared with the industry standard (Xsens) (Grace Gaerlan et al., 2012, Lee et al., 2013). This work uses a method developed by (Mayagoitia et al., 2002) to asses postural sway using only a 3-axis accelerometer to asses motion of sway.

Xsens are set of sensors used to capture motion data and are commonly used for research application in physical therapy Grace Gaerlan et al. (2012), Lee et al. (2013). This commercially available system could provide a good benchmark when comparing against the custom built sensor. This work examines the performance of a custom-built sensor built around an ICM-20948 and attempts to compare performance characteristics. ICM-20948 is an all in one 9-axis sensor with a 3-axis: gyroscope, accelerometer, and compass. Understanding the performance characteristics for postural sway should provide a reasonable threshold of performance.

Understanding the process required to build an IMU along with the tools required can be of use for applications outside the bare minimum of collecting and storing IMU data and aid the assessment of data in other applications. The process is similar for use with other processors and other IMU units on the market. 


\subsection{Specific Aims}

1.1.1 Aim\#1 Develop a wearable sensor capable of measuring postural sway with off the shelf components.

- Ardunio is a good starting point with a large set of libraries and tools for prototyping

- Write firmware that collects data from an onboard IMU and stores it in a format that can be later analyzed

- Develop a tool that converts the data into a format that can be used for analysis, for example *.csv format files

1.1.2 Aim\#2 Generate Understand Periodic movement in controlled environment between tracking marker and IMU.

- known period movement in a controlled environment

- verify performance of IMU with marker as ground truth

1.1.3 Aim\#3 Utilize the sensor with a healthy population with different proprioceptive and vibration conditions and identify problems.

- Verify the performance of the sensor with a set of healthy subjects with a set of verifiable conditions

- verify the sensor with Xsens as a ground truth

- parameterize the data into different factors and run a model that examines cross effects for the set of given conditions. 


\subsection{Organization}

This thesis has 5 chapters. Chapter 1 covers information about the background of the study along with aims and objectives. Chapter 2 covers the design of the IMU boards along with components where they were assembled, firmware along with the tools used to build the firmware, how the data was stored and additional software that was used to decode the data. Chapter 3 covers procedures to capture student collected data along with methods to parameterize the raw time series data, explanations and analysis. Chapter 4 works on creating a sythetic setup to verify the validity of a motion sensor for use in identifying if the signal originates from a common source and validity of the source signal. ?? concludes the work of this thesis. 


\section{CHAPTER 2}

\section{Hardware}

\subsection{Mini Data Logger}

\subsubsection{Introduction}

Arduino is a powerful platform with a large selection of libraries and easy to use API's that allow for easy prototyping. The platform and tools are all open source which offers a lot information about the platform including compiling the source code along with schematics and layouts. It's a fairly easy process to go from protype to a board with similar components.

\subsection{Materials and Methods}

\subsubsection{Hardware}

This sensor uses an ICM-20948 and an $\mathrm{I}^{2} \mathrm{C}$ bridge PCA9306DCUR. The recommended schematic from the datasheet is used to inform the design of the final circuit board; including the MEMS 2.2 and microprocessor. The mems sensor runs at $1.8 \mathrm{v}$, which is lower than the $3.3 \mathrm{v}$ for the ATmega processor. A separate drop regulator is used to bring the voltage down to the required $1.8 \mathrm{v}$ 


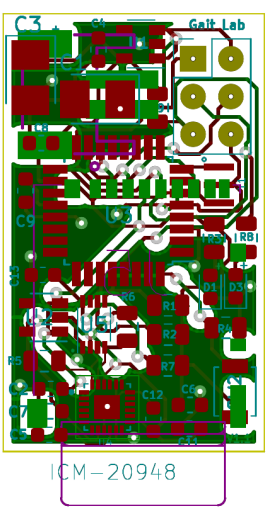

(a) Circuit Ki-CAD Design

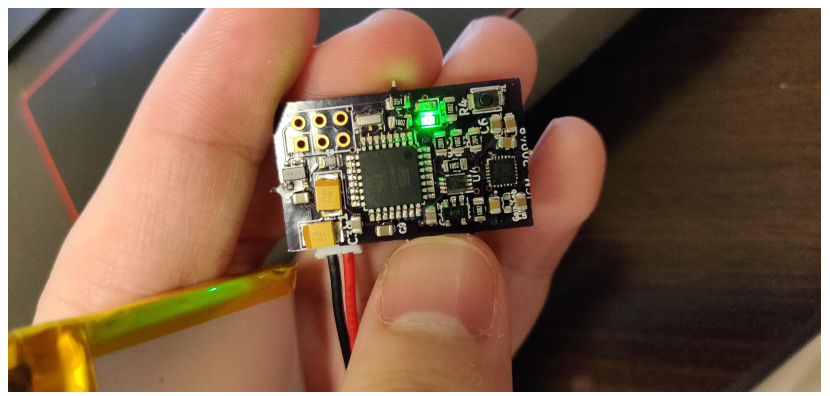

(b) Fabricated Circuit

Figure 2.1: Mini-Logger

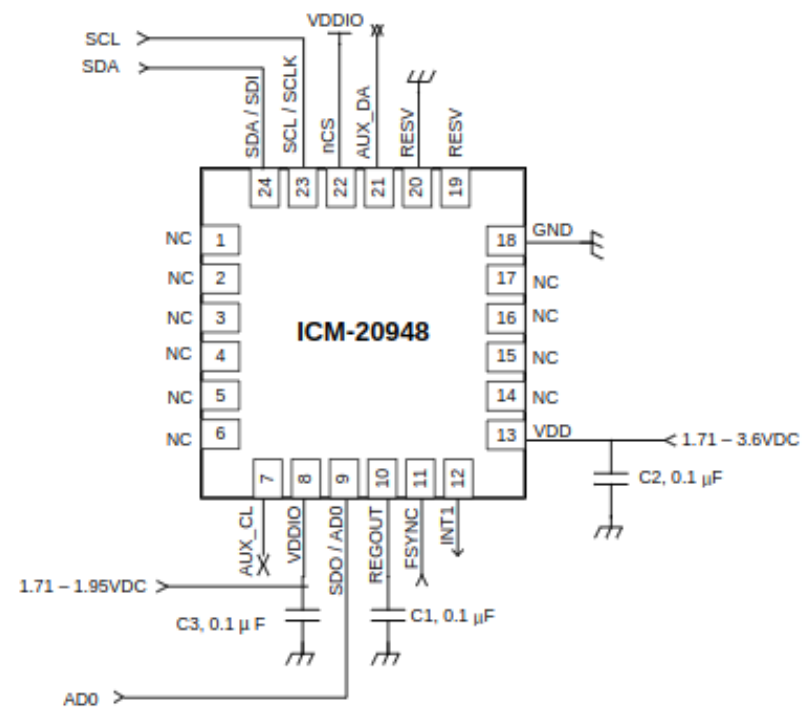

Figure 2.2: ICM-20948 recommend schematic from datasheet (ICM-20948) 
(TLV70218). This voltage is much lower then other mems developed by TDK. The board was powered with an external li-poly battery connected with a JS2-2 connector.

The two major interface protocols between Integrated Components (IC) are the $\mathrm{I}^{2} \mathrm{C}$ bus specification and Serial Peripheral Interface (SPI). These two are mainly used to talk between other processors and embedded peripherals. $\mathrm{I}^{2} \mathrm{C}$ is a two-wire protocol used in a master-slave configuration where the same two wires can be connected to multiple slave devices. A slave address is given to each device on the pair so only one slave device can talk with the master at a time. SPI is a three-wire protocol, and a fourth wire is used to select multiple devices on the same line. SPI is a dual-duplex where data can be sent in both directions where $\mathrm{I}^{2} \mathrm{C}$ is handled in turn. Each additional device introduces an extra wire for the selection. Two devices use 5 wires; three devices use 6 so on and so forth.

The ICM-20948 is configured for $\mathrm{I}^{2} \mathrm{C}$, but there are also options for SPI. SPI supports higher sample rates up to $7 \mathrm{Mhz}$ while $\mathrm{I}^{2} \mathrm{C}$ supports data rates up to $400 \mathrm{kHz}$ but only in high-speed mode. For this application, these higher data rates are not necessary since $500 \mathrm{~Hz}$ is well below this $400 \mathrm{khz}$ range. $\mathrm{AD} 0$ determines the slave address of the device, which can be $0 \times 68$ for low and 0x69 for high. Section 4.2 from the datasheet shows the typical operating circuit for the IMU for both $\mathrm{I}^{2} \mathrm{C}(2.2)$ and SPI operation.

The SD card has two modes of configuration, SD mode, and a slower SPI mode. For this case, the slower SPI mode is more than enough bandwidth for the ATmega processor. SdFat is an Arduino library that implements the API for writing and reading files to and from the SD card. Micro SD is an 8 pin device. The pin-outs for the SD card can be found in figure 2.3. In SPI mode, one bit is written at a time where SD mode has a bit width of up to 3 bits for micro SD and 4 bits for standard SD (SD Specification).

\subsubsection{Firmware}

The firmware was written in $\mathrm{C}++$ and compiled with the avr-gec and avr-g++. Scons was used to setup the environment and compile the source for the Atemga. The process is similar to writing 


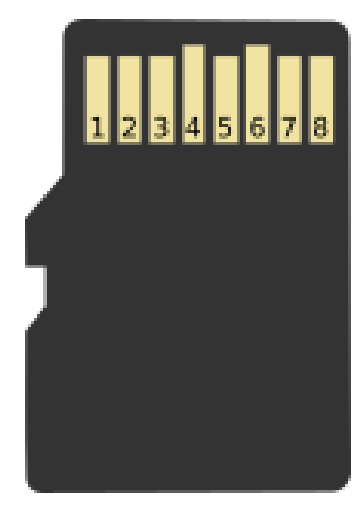

\begin{tabular}{|l|l|l|}
\hline Pin\# & Name & Description \\
\hline 1 & Dat2 & X \\
\hline 2 & CD/Dat3 & Chip Select (neg true) \\
\hline 3 & CMD & Data In/MOSI \\
\hline 4 & VDD & Supply Voltage \\
\hline 5 & CLK & Clock \\
\hline 6 & VSS & Supply Voltage Ground \\
\hline 7 & DAT0 & Data Out/MISO \\
\hline 8 & DAT1 & X \\
\hline
\end{tabular}

Figure 2.3: SD card pinout

Ino files using the Ardunio IDE which use a fairly similar set of tools (avr-gcc, avr-g++, and avrdude) that build and install the ardunio code. The final elf binary is converted to Ihex (Intel Hex) and flash to the processor with Avrdude using an Atmel Ice Programmer.

A $2 \times 3$ ISP header is left exposed and was programmed with a pogo pin adapter. Additional bits have to be set along with flashing the firmware. These fuse bits disable and enable features built into the processor. Some of these can be configured in code while others have to be set by the programmer. A fuse calculator can be used to set the correct fuses for the flash utility. This includes changing the internal clock to an $8 \mathrm{Mhz}$ external clock and disabling div by 8 for the clock rate. The compiled source expects $8 \mathrm{Mhz}$ and this can be configured with Microcore by setting 'CPU_FREQUENCY' to the desired sample frequency; in this case, it would be 8 million times a second or 1 million if divide by 8 is set.

The option to flash the processor over the UART interface is not possible because the Arduino bootloader is not configured with the ATMEGA processor. The Ardunio bootloader is a small chunk of code at the start of the memory and allows for the ability to program the ATMEGA processor through UART. This also requires a crystal because the timing over UART is much more precise. Protocols such as SPI and $\mathrm{I}^{2} \mathrm{C}$ use a separate clock line to keep time with transferring data.

The external push button on the board starts a session and clicking it again stops it. Each new session produces a new bin file. When the processor is not logging data, the processor is placed into a sleep state, along with the IMU. For efficiency, a large file is allocated on the SD card 
before writing. Write efficiency is improved if the data is written block by block. The set of samples are cached and written all at once to each 512 block sector. Each payload starts with the number of samples in the block then an array of samples. Each sample stores the 3-axis accelerometer, 3-axis gyroscope, epoch timestamp and temperature. The payload consists of first the number of entries in that chunk of data then an array of samples and finally empty bytes fill in anything that does not fully occupy the block. Each write cycle writes 512 bytes. This setup avoids corruption because a failed write does not affect more than a single block. It is relatively simple to read back from the SD card and skip blocks that have been corrupted due to a failed write.

The ICM-20948 is configured through a set of readable and writable registers. A fixed register is used to switch between different set of registers organized into four banks. Each register has a 1-byte address and 1 byte of writable/readable memory. REG_BANK_SEL is used to change the register bank. PWR_MGMT_1 is used to place the device into sleep and also wake up the device when the sensors are not enabled. ACCEL_CONFIG and GYRO_CONFIG are used to configure the gyroscope and accelerometer. These are the key registers, but there are a lot more configuration options that could be explored.

The sensor only provides the data for 6-axes (accelerometer, gyroscope). The data is stored in a 2 byte signed short which provides a full resolution from $-32,768$ to 32,767 . The range can be exchanged for sensitivity by setting ACCEL_FS which supports: \pm 2 G's, \pm 4 G's, \pm 8 G's, \pm 16 G's. This involves dividing the raw value by a scaling factor. Using one range limits the resolution to the scaled range and saturates if the number of G's exceeds that target sensitivity. The resolution chosen was $\pm 4 \mathrm{G}$ 's where the act of standing would not exceed this fixed range but offers higher sensitivity. 
Sensitivity ACCEL_FS

\begin{tabular}{|l|l|}
\hline \pm 2 & 16,384 \\
\hline \pm 4 & 8,192 \\
\hline \pm 8 & 4,096 \\
\hline \pm 16 & 2,048 \\
\hline
\end{tabular}

\subsubsection{Software - Data Decoding}

A separate application was written to decode the raw data collected from the SD card. The application was written in QT; A C++ graphical toolkit library. The serialized data is stored in chunks of 512 bytes as described from the previous section. So decoding the data involves reading chunk by chunk and extracting the relevant signals from each chunk. A 512 block chunk starts with 16 bit unsigned short describing the number of entries and another 16 bit unsigned short for the number of times that data overran the current block. Anything after the 32 bits is the raw data collected from the sensor up to the last possible sample that can be stored in that chunk. each sample is 18 bytes long where: 4 bytes (time), 2 bytes(acc_x), 2 bytes(acc_y), 2 bytes(acc_z), 2 bytes(gyro_x), 2 bytes(gyro_y), 2 bytes(gyro_z), 2 bytes(temperature). For each chunk, the max number of samples that can be stored in a sample is exactly 28 . This leaves only 4 bytes that are padded as zeros, so each chunk is exactly 512 bytes.

\subsection{Results}

The Mini Logger board is based off an Arduino Pro mini 3.3v at 8Mhz. The layout of the board is designed with Kicad and exported to Gerber files. A separate CSV (comma separated value file) was created, mapping the components to the associated footprints. Wherever possible, components are sourced from Seeds OPL (Open Parts Library) else they are sourced from either Digikey or Mouser. The final board is assembled from Seeed Studio. The firmware was compiled against MiniCore, a derivative Ardunio kernel. Libraries written for Arduino core should function the same when compiled against MiniCore. Minicore is a general library that supports 
other processors in the same family including ATmega328, ATmega168, ATmega88, ATmega48, ATmega8 and all variants of A, P, PA, and PB (ATMEGA).

\subsection{Discussion}

There are other features for the ICM-20948 that can be tweaked to improve performance characteristics such as using the low band pass filter that comes with the IMU given that the the bandpass filter on the IMU can sample from a much higher sample rate then after the fact. Generally the performance can be improved by augmenting the data with other signals such as a pressure sensor, compass, or Magnetometer. the IMU can be paired with AK09916(magnetometer) and this should in theory also improve performance of the IMU. Atmega328 only has $32 \mathrm{~kb}$ of space and a fairly low end $8 \mathrm{Mhz}$ processor. the process of reading the writing the data to the SD card was pushing the limits of the Atmega processor and a better alternative would be needed.

The Atmega platform is also fairly limited and heavily handy capped by limited amount of processing and memory. It is possible to do the same task with fewer instructions which both improves execution speed and also shrinks the footprint of the program. This requires modifying registers directly which requires a fairly strong understanding of the AVR platform, but other platforms exist for low energy applications with more resources and better tooling. The ARM platform has grown a lot in the last couple of years especially for wearable. System of Chip (SOC) are single form factor units which can provide both the processor and a secondary system such as bluetooth.

\subsection{Conclusion}

The platform built around the sensor has several limitations. The process of pressing a tactile button introduce noise into the data and extracting the SD card and saving the data introduces extra human error. Some more work is necessary to correct some of these flaws in the protocol. The protocol and process for developing such a sensor is provided as a good starting point and should inform future work that attempts to improve on this model. There is a lot of space for improve- 
ment along with different methods that should produce a better outcome. More work will have to done to examine other configurations that would better appropriate the IMU. Other methods can be combined to improve the characteristic performance of the IMU.

There are other features for the ICM-20948 that can be tweaked to improve performance characteristics such as using the low band pass filter that comes with the IMU given that the the bandpass filter on the IMU can sample from a much higher sample rate then after the fact. The bare silicon of the IMU will perform the task more efficiently then the equivalent code executed on the processor.

Generally the performance of an IMU can be improved by augmenting the data with another signal such as a pressure sensor, compass, or Magnetometer. the IMU can be paired with AK09916(magnetomet and this should in theory also improve performance of the IMU. Atmega328 only has $32 \mathrm{~kb}$ of space and a fairly low end $8 \mathrm{Mhz}$ processor. the process of reading the writing the data to the SD card was pushing the limits of the Atmega processor and a better alternative would be needed. 


\section{CHAPTER 3}

\section{Postural Sway Assessment from Inertial Sensors With Human Subjects}

\subsection{Introduction}

Human Postural is maintained through three important systems: visual, vestibular, and Somatosensory sensory systems (Peterka, 2002). These systems degrade with age and individual become more prone to falling (Grace Gaerlan et al., 2012).

Visual information plays a role in perception of motion. The central (focal) specializes in motion detection and motion recognition (Grace Gaerlan et al., 2012). Peripheral vision helps with understanding displacement and is used as feedback to control sway (Grace Gaerlan et al., 2012). For Eyes open and eyes closed condition, participants were either asked to stare at a fixed point or blind folded to remove visual information. It is known that individuals that have an impaired Vestibular or Somatosensory system rely more on visual information (Guerraz \& Bronstein, 2008).

Vestibular balance input is maintained through the inner ear and motion if identified through deviation of head orientation where as visual information work out head position through visual cues (Peterka, 2002). Histologic reports have shown significant hair cell decline with age (Za- 
lewski, 2015). This decline in cell loss impacts the ability of the vestibular system to detect motion.

Proprioceptive provides information about muscle length and velocity through mechanoreceptor (Shaffer \& Harrison, 2007, Grace Gaerlan et al., 2012). This helps with discerning the location of joints, along with providing a positional sense of the environment. Muscle fibers play an essential role in more reflexive and voluntary movements informed by the proprioception system (Shaffer \& Harrison, 2007). Balance information is provided through the plantar surface of the foot, and is main point of contact between the ground and the body (Ekvall Hansson et al., 2019). The force applied to the sole is non-uniform and provides sensory cues that enhance postural response. StepRight stability trainers were used to disrupt sensory information from the ground and plantar surface of the foot. This forces students to rely on vestibular information and visual information to maintain balance. For some of the trials, participants had their eye's closed, so they had to rely more on vestibular information.

Proprioceptive can be enhanced with the use of sub-sensory noise. Several studies have verified that introducing noise to a sensor motor system for either static or dynamic tasks can improve performance of that task (Costa et al., 2007, Priplata et al., 2006). A non-zero amount of noise applied to a sensory system may significantly affect an individuals ability to feel weak sensory stimuli through a process called stochastic resonance (SR) (Martínez et al., 2007). SR is an affect introduced into nonlinear system where external noise is introduced into a system. Noise added in an optimal amount enhances information transfer. It has been demonstrated that SR applied to the feet has been shown to improve postural stability.

Sub-sensory noisy was introduced through the use of a C2 tactor. A typical design for a vibrating motor uses an eccentric mass that is driven by a small de motor (Yao \& Hayward, 2010). The amplitude and frequency are linked; This is influenced by the weight and rotational speed of the weighted mass (Yao \& Hayward, 2010). The typical response of a motor like this involves a ramp where the motors are driven to speed. This is very problematic, when a precise response is needed (Yao \& Hayward, 2010). The general design of a tactor uses similar to a traditional 
speaker with a cylindrical magnet suspended by two rubber membranes and a voice coil that drives the magnet and diaphragm (Yao \& Hayward, 2010). These devices offer frequencies in the range of $0-500 \mathrm{~Hz}$ and are ideal for the task of Stochastic Resonance response (Yao \& Hayward, 2010).

\subsection{Materials and Methods}

Data was collected from both the mini_logger and Xsens. Both acceleration units were mounted on the T2 spine position as shown in figure 3.2. Each data collection session was 1 minute long, and individual performing quiet standing each trial consisted of 10 different random treatments given to each applicant for each trial. The different conditions are listed in table 3.1. The data was collected over three sessions and consisted of 10 healthy adults ( 5 males and 5 females, age: $27.3 \pm 2.7$ years). They were recruited and approved Chapman University IRB. Subjects provided informed consent as per Chapman University IRB and were evaluated on ability to maintain balance with eyes open and with eyes closed, intact sensation of the foot, and intact proprioception of the ankle. Subjects were excluded if they presented with foot pain while barefoot or required orthotics. Each trial consisted of 12 possible conditions listed in table 3.1 were picked randomly. Students were asked to stand in place for 1 minute and undergo one of the 12 possible conditions. All trials were randomized and subjects were not aware of the treatment they would receive when performing the trial. None of the student subjects had a history of vestibular or balance disorders problems related to performing the study. The goal of this study is to investigate if IMU's can identify the different conditions and characterize those signals correctly.

The table 3.1 show all the possible combinations for each treatment. The treatments include use of tactors, $260 \mathrm{~Hz}$, and $280 \mathrm{~Hz}$. For the tactor trials, a tactor was attached to the first metatarsal and the frequency was changed in order to see if the balance of the student would change. Students kept the tactors mounted so they were not aware when the tactors were active or not this can be seen between figure 3.2c and figure 3.2b. For the trials with an uneven surface, a StepRight system was used to lower the points of contact of the foot. Eyes condition determines if the indi- 


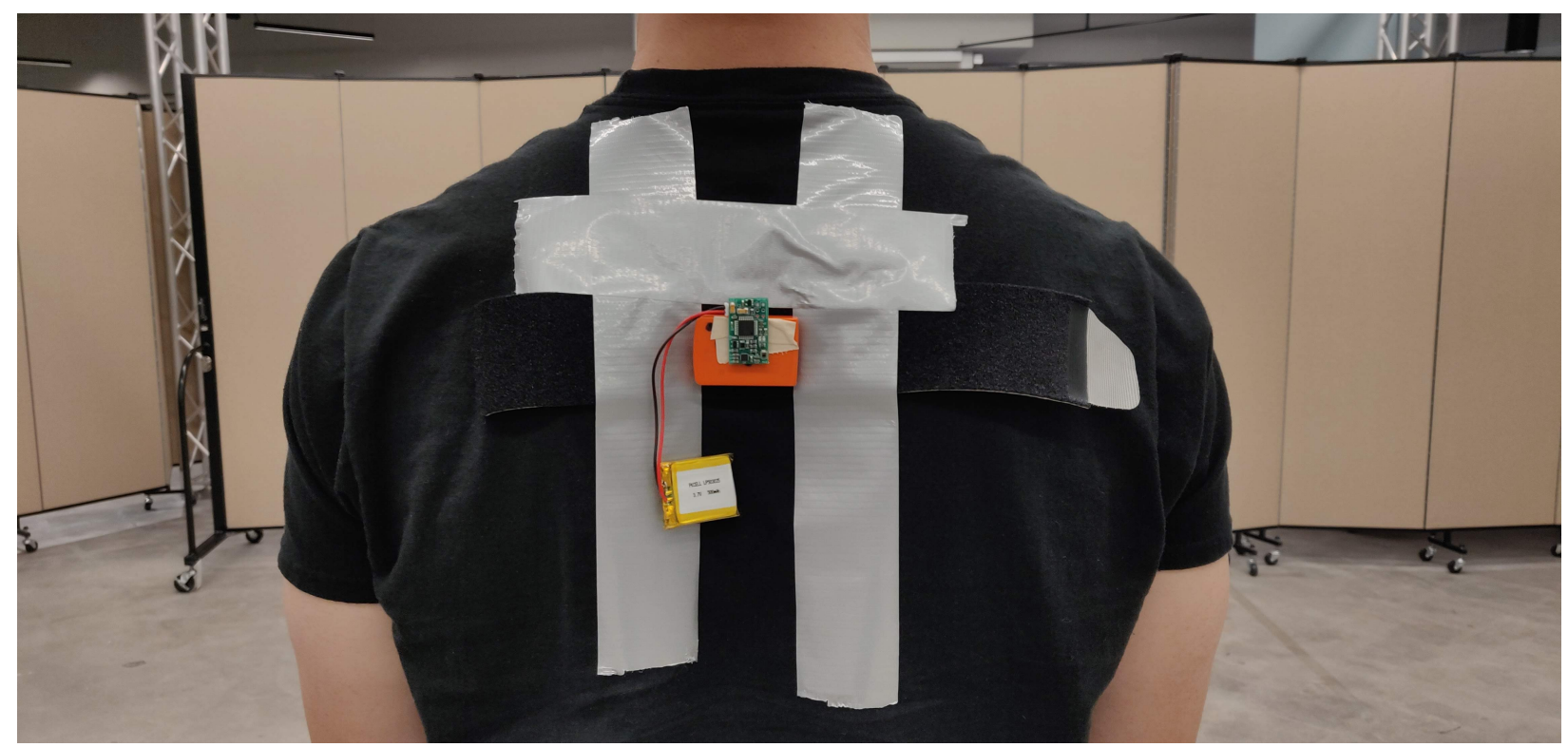

Figure 3.1: Logger Mounting

vidual in blindfolded for that trial.

\begin{tabular}{|l|l|l|l|}
\hline Condition & Tactor & Eyes Condition & Somatosensory Condition \\
\hline C1 & $0 \mathrm{~Hz}$ & EO & Even \\
\hline C2 & $0 \mathrm{~Hz}$ & EO & Uneven \\
\hline C3 & $0 \mathrm{~Hz}$ & EC & Even \\
\hline C4 & $0 \mathrm{~Hz}$ & EC & Uneven \\
\hline C5 & $260 \mathrm{~Hz}$ & EO & Even \\
\hline C6 & $260 \mathrm{~Hz}$ & EO & Uneven \\
\hline C7 & $260 \mathrm{~Hz}$ & EC & Even \\
\hline C8 & $260 \mathrm{~Hz}$ & EC & Uneven \\
\hline C9 & $280 \mathrm{~Hz}$ & EO & Even \\
\hline C10 & $280 \mathrm{~Hz}$ & EO & Uneven \\
\hline C11 & $280 \mathrm{~Hz}$ & EC & Even \\
\hline C12 & $280 \mathrm{~Hz}$ & EC & Uneven \\
\hline
\end{tabular}

Table 3.1: Different conditions of postural standing 


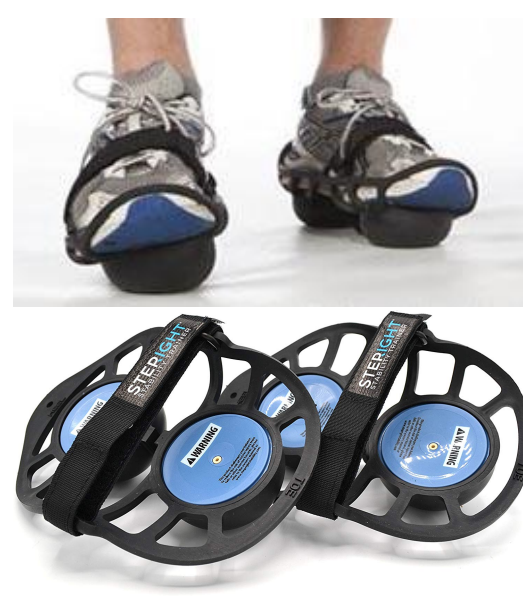

(a) Steprights

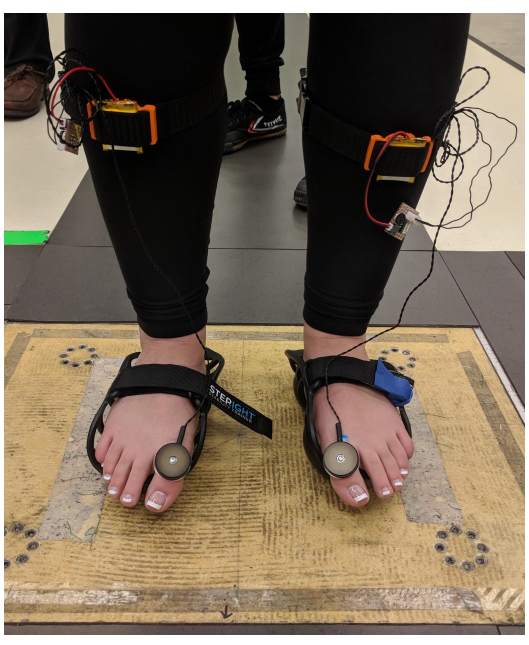

(b) With Stepright and tactor mounting mounting

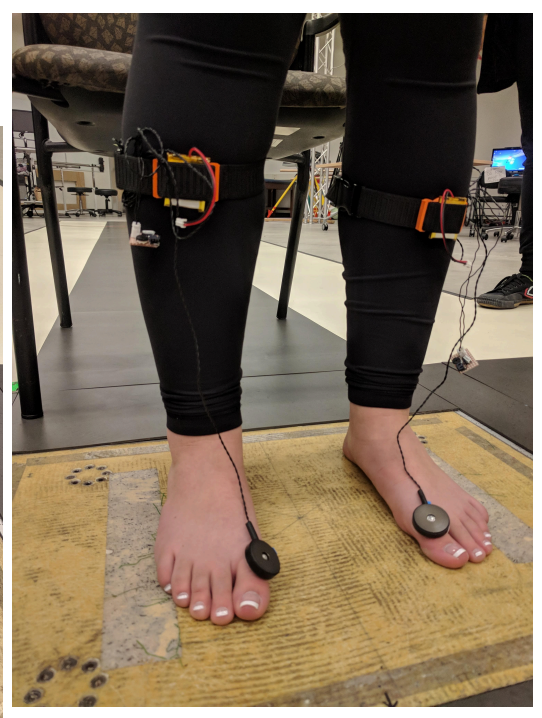

(c) Without Stepright and tactor

Figure 3.2: Experimental Setup

\subsubsection{Converting Raw Acceleration data to Center of Pressure}

the standing balance of an IMU uses a method developed by Mayagoitia et al. (2002) Where the vertical gravity vector is used to work out the orientation of the sensor. The IMU is placed at T2 each individual. The relative change in the acceleration due to the rotation of the device in comparison to static gravity is used to project a point down on the ground. $a_{x}, a_{y}$, and $a_{z}$ are axes provided from the accelerometer. The magnitude of the resultant is given as $\mathbf{R}(\mathrm{mm} / \mathrm{s})^{2}$, and the directional angles are described from the resultant from the equation 3.1.

The accelerometer may slightly be tilted due to the curvature and posture of the standing individual. Depending on the placement, the sensor can be close or farther from the ideal vertical axis. The off-axis tilt of the sensor has to be corrected to line up with a horizontal coordinate system. This is calculated by using the average tilt of the sensor and applying a fixed angle transformation (Mayagoitia et al., 2002, Moe-Nilssen, 1998b,a).

The values generated by the IMU are not comparable to a forceplate since the methods collected are vastly different. This makes a couple assumptions such as the height of the individual and ignores acceleration affects that will exaggerate the tilt. The tilt of the sensor on T2 will also 
stretch the projected surface.

$$
\begin{gathered}
R=\sqrt{x^{2}+y^{2}+z^{2}} \\
\cos (\alpha)=\frac{a_{x}}{R} \quad \cos (\beta)=\frac{a_{y}}{R} \quad \cos (\gamma)=\frac{a_{z}}{R}
\end{gathered}
$$

The relative projected location based off the sensors are then described by the equations from 3.3. $d_{z}$ is the relative height of the sensor off the ground. The relative height is unknown so a fixed estimate based off of the average height of an adult human is used in this case. This is a relative metric and is thus not directly comparable to a pressure plate. $d_{x}$ is the projected point on the ground relative to the Anterior/Posterior and $d_{z}$ is projected point on the medial/lateral. This follows the assumption that the swept angle change by the accelerometer is small and the distance from the accelerometer and the ground is constant.

$$
D=-\frac{d_{z}}{\cos (\gamma)} \quad d_{x}=D * \cos (\beta) \quad d_{z}=D * \cos (\alpha)
$$

\subsubsection{Driver Board Assembly}

A small driver board was assembled for each tactors, and produced a signal at a fixed square wave frequency from $0 \mathrm{v}-4.7 \mathrm{v}$. The $\mathrm{C} 2$ tactor is an open voice coil type transducer (Yao \& Hayward, 2010). The contactor can be placed against the skin for a more localized tactile sensation. For this use case, the backside of the tactor was placed against the subjects foot where the vibration reverberates through the metal casting. The vibrations are adjusted to a point where the subject was unaware if they were given the treatment or not. One key feature of applying the signal at a sub-threshold means the subjects were not aware they were being given the treatment or not. Testing these noises at sub-threshold rules out confounding factors where the subject gives more attention to the localized tactile sensation (Severini \& Delahunt, 2018). SR was tested with a control condition where no signal is provide, $260 \mathrm{~Hz}$ and $280 \mathrm{~Hz}$. 


\subsubsection{Calculation Parameters}

\section{Elliptical Area}

The method that was used to find the elliptical area within a fixed confidence interval as described by (Schubert \& Kirchner, 2014). The area $A_{E}$ of an ellipse is calculated from the major and minor axes of the ellipse (Schubert \& Kirchner, 2014). This can be seen from equation 3.4. The goal is to calculate the area of the ellipse that best represents the sampled data within a given confidence interval.

$$
A_{E}=\pi a b
$$

PCA is applied to reduce the complexity of the data down to its principle components. The process of finding the correct values for the major axes involves finding Eigenvalues $\lambda_{1}$ and $\lambda_{2}$ from the sample covariance matrix. This can be described as $|A-\lambda I|$ where $\mathrm{I}$ is the identity. The Eigenvalues can be found from the equation 3.5.

$$
\lambda_{1}=\frac{1}{2}\left[s_{x}^{2}+s_{y}^{2}+\sqrt{\left(s_{x}^{2}-s_{y}^{2}\right)^{2}+4_{x y}^{2}}\right] \quad \lambda_{2}=\frac{1}{2}\left[s_{x}^{2}+s_{y}^{2}-\sqrt{\left(s_{x}^{2}-s_{y}^{2}\right)^{2}+4_{x y}^{2}}\right]
$$

Final major and minor axis values are described by 3.6. This is a bi-variate area that covers $100(1-\alpha) \%$ probability of future observations. Each axe is described by Hotelling T-squared distribution where a large value of $\mathrm{n}$ approaches the chi-squared distribution as described by 3.6 (Schubert \& Kirchner, 2014). This region forms an elliptical mound where the counters of the mound describe different confidence intervals. We used an $\alpha$ value of $.95, .85$, and .99 of coverage when evaluating the performance of the sensor in the student collected data.

$$
a_{p}=\sqrt{\chi_{2}^{2} \lambda_{1}} \quad b_{p}=\sqrt{\chi_{1}^{2} \lambda_{2}}
$$




\section{Circular Area}

The circular area 3.7 is a circular area that is based on the average distance from the mean to every sample point in the sample set. The area of coverage uses the area of a $\operatorname{circle}\left(\pi r^{2}\right)$ to get the coverage of sway.

$$
c_{a}=\pi r^{2}
$$

\section{Path Length}

The path length is calculated by summing up the individual segments from the projected path. Postural velocity is calculated by dividing the total length of the path by the total time and average velocity is calculated by diving the length of travel by a fixed cutoff time. Postual velocity isn't too useful since the samples were all collected with the same amount of time. The average velocity is just a scalar amount based off the total distance.

$$
P_{\text {length }}=\sum_{k=2}^{N} \sqrt{\left(x_{k}-x_{k-1}\right)^{2}+\left(y_{k}-y_{k-1}\right)^{2}} \quad P_{v}=P_{\text {length }} / T
$$

\section{Power Frequency}

Mean power frequency (MNF) is an average frequency, calculated as the sum of products of both the power and frequency over the total power spectrum (Oskoei \& Hu, 2008, Phinyomark et al., 2013). The definition of the function is described by the equation 3.9. $f_{j}$ is the frequency value of the spectrum for the given bin $\mathrm{j}$ and $P_{j}$ in the power spectrum.

$$
M N F=\frac{\sum_{j=1}^{M} f_{j} P_{j}}{\sum_{j=1}^{M} P_{j}}
$$

Max power frequency describes the dominant frequency in the signal. The dominant frequency is the index $\mathrm{j}$ index from the max power in the power spectrum when mapped against frequency. 
This is applied for AP, ML, and the scalar value of the AP and ML values. The power and frequency spectrum is calculated using Scipy welches function with a segment length of 1205.

Root Mean Squared

$$
x_{r m s}=\sqrt{\frac{1}{N} \sum_{j=1}^{N} x_{j}^{2}}
$$

RMS or quadratic means is the square root of the mean value of $x_{j}^{2}$. This is applied for AP, ML, and the scalar value of the AP and ML values. RMS is used to calculated the dispersion of data relative to the zero (Moe-Nilssen, 1998b). The average is influenced by negative values in the set and lower the effective scale of numbers. RMS can describe the relative scale of the set regardless if the value is positive or negative. This is good in characterizing the relative scale of motion when describing COP.

Data was collected from both the Xsens and mini-logger. The IMU data was collected at a rate of $500 \mathrm{hz}$ from the mini-logger and $100 \mathrm{~Hz}$ from the Xsens sensor. The mini-logger was mounted on top of the Xsens so both sets of data can be comparable. The data is first filtered with a 3rd order low-pass Butterworth filter at $8 \mathrm{~Hz}$ cutoff frequency. The action of Pressing the button on the mini logger produces a large spike on the acceleration so 1 second of the data is shaved off the beginning of each set. For compatibility, the data was re sampled to $100 \mathrm{~Hz}$ for the mini-logger. The filtering for both sensors can be seen for Xsens for figure 3.3a and the mini logger for figure 3.3b. The mini logger has a lot more high-frequency noise when compared to the Xsens sensors but this could be addressed by enabling the low pass filter that comes with the ICM-20948. The final data from the mini-logger was resampled with fourier method so the final data matches up with the xsens sensor. 


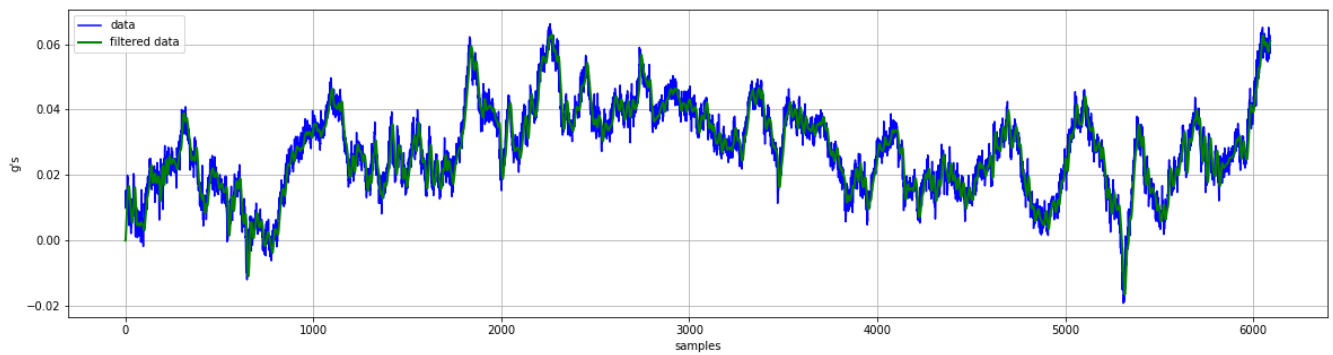

(a) Filtered Xesns Data

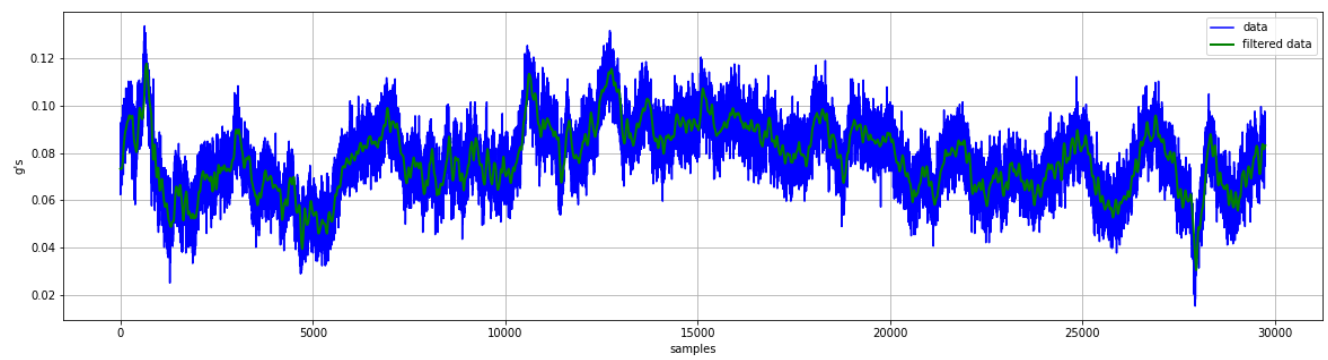

(b) Filtered Mini Logger Data

Figure 3.3: Filtered IMU Data

\subsubsection{Parameters}

The engineered parameters included; 95_EllipticalArea, 99_EllipticalArea, RMS_COP, RMS_COP_ML, RMS_COP_AP, SwayVelocity, CircularArea, DominantFreq_COP_R, DominantFreq_COP_ML, DominantFreq_COP_AP, MeanFreq_COP_R, MeanFreq_COP_AP, and MeanFreq_COP_ML. MANOVA analysis was run on the data using JMP pro software with a significance level with a $p<0.05$ for all analyses. Interaction affects related to the instrument are of particular interest because in these cases the performance of the sensor can influence the outcome of the results.

\subsection{Result}

RMS_COP is significant between mini_logger and Xsens and shows that the variance for the mini_logger is much higher then xsens. We found that the mean frequency for both AP and ML was much lower for mini_logger.

An interaction between SwayVelocity was significant with a difference $(p<0.0001)$ when 
looking at sway velocity relative to eye condition. Specifically, there was the most significant difference between EC UES $(\bar{x}=0.0218)$ and EC ES $(\bar{x}=0.008)$.

SwayPath length was also a factory with a significant difference $(\mathrm{p}<0.001)$ when looking a sway path length relative to eye condition. Specifically, there was a significant difference between $\operatorname{EC~UES~}(\bar{x}=0.8745)$ and $\operatorname{EC~ES~}(\bar{x}=0.32022)$.

we found RMS of signals when using StepRight (reduced proprioceptive conditions) at $0 \mathrm{~Hz}$ $(\bar{x}=0.035)$ were significantly higher $(\mathrm{p}=0.0155)$ when compared to the condition when using StepRights at $200 \mathrm{~Hz}(\bar{x}=0.026)$. However, no differences were observed at $260 \mathrm{~Hz}$.
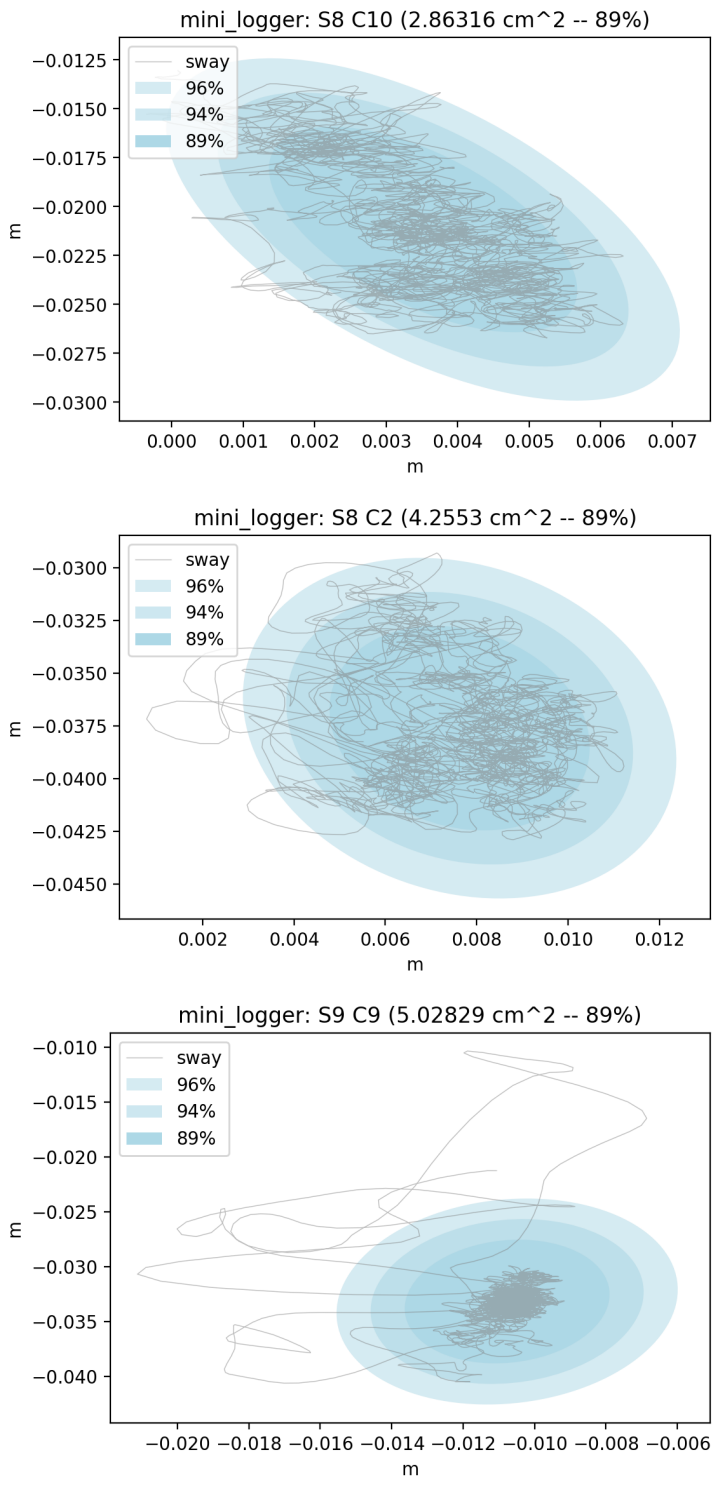
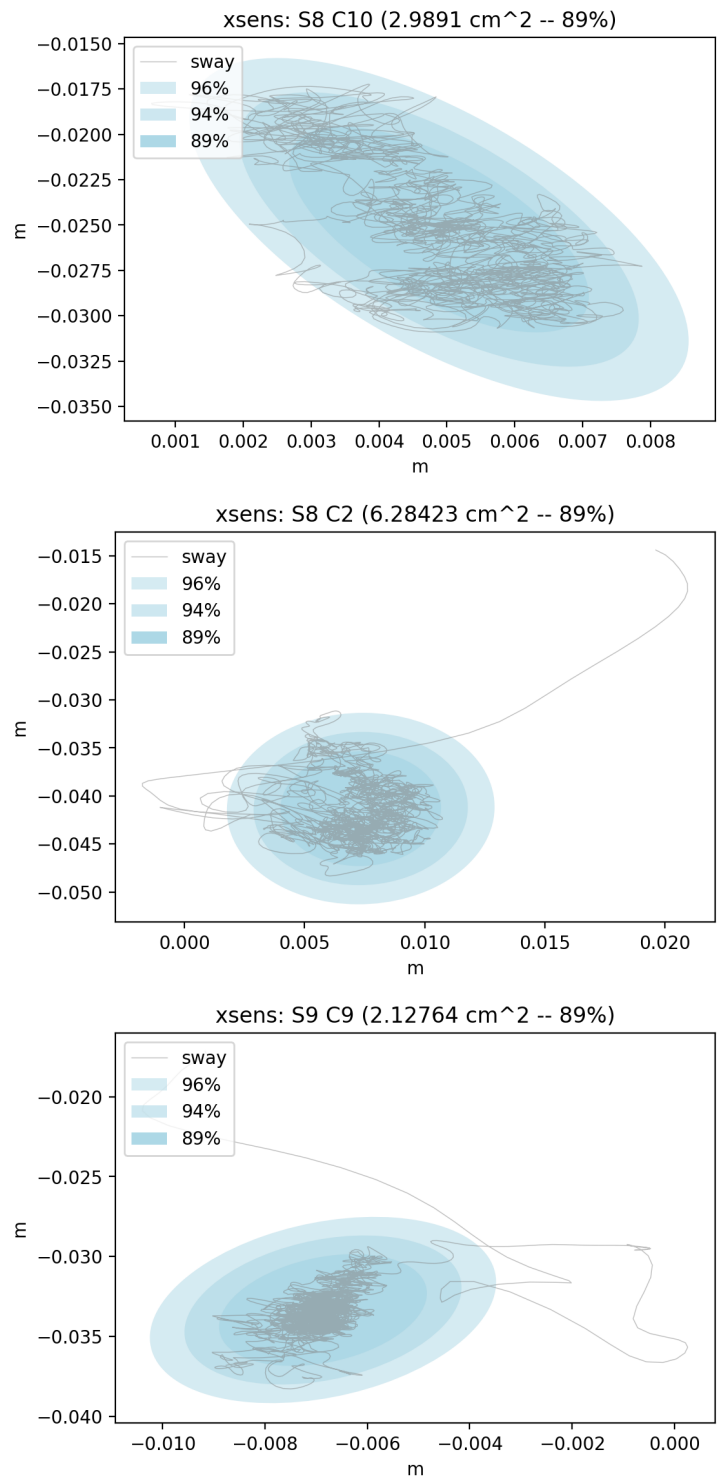

Figure 3.4: Comparison of mini logger and xsens for a given trial. 


\subsection{Discussion}

To identify if wearable sensors are sensitive to varying proprioceptive, tactile inputs and visual inputs. A combination of 12 postural conditions were tested among 10 healthy subjects as shown in Table 3.1. This study investigates the effect of various frequencies of stochastic resonance on the postural sway of healthy young adults during quiet stance under conditions challenging their three sensory systems.

Relying on just the acceleromter for the orientation of the sensor is not accurate in some cases. The starting orientation of the IMU can heavily skew the data and correcting the orientation with the average tilt is only a rough approximation. This method would be a lot better if this accounted for a change in rotation from the gyroscope to correct for the change in tilt.

A small trial with subjects can verify the validity of the sensor along with identifying problems with the setup and inform future works that builds on the existing setup. A synthetic step is necessary to work out the validity of the setup and verify feasibility of the setup. Generally the built part performs worse then the industrial standard but the sensor just has to perform well enough to be able to identify the root cause at a significantly reduced price point.

\subsection{Conclusion}

We found that postural sway, as represented by RMS COP, increased across all conditions when subjects were instructed to stand on StepRights (a condition with decreased proprioceptive inputs), compromising somatosensory information from the ground. However, our results revealed that stochastic resonance during these somatosensory-compromised conditions improved postural sway compared to control groups without vibratory input. These results are in agreement with

previous research examining the effects of stochastic resonance on postural sway (Bagherzadeh Cham et al., 2016, Cloutier et al., 2009, Bagherzadeh Cham et al., 2016, Postema et al., 2009, Costa et al., 2007, Wei et al., 2012). We found significant improvements in COP measurements at vibration frequency of $220 \mathrm{~Hz}$. Whereas no difference was observed between control trials and tri- 


\begin{tabular}{|c|c|c|c|c|c|c|c|c|c|c|c|c|c|}
\hline \multicolumn{14}{|c|}{ Mini Logger } \\
\hline \multicolumn{14}{|c|}{ Eyes Condition } \\
\hline & & \multicolumn{6}{|c|}{ Eyes Closed } & \multicolumn{6}{|c|}{ Eyes Open } \\
\hline & & \multicolumn{6}{|c|}{ Somatosensory Condition } & \multicolumn{6}{|c|}{ Somatosensory Condition } \\
\hline & & \multicolumn{3}{|c|}{ Control } & \multicolumn{3}{|c|}{ StepRight } & \multicolumn{3}{|c|}{ Control } & \multicolumn{3}{|c|}{ StepRight } \\
\hline & & \multicolumn{3}{|c|}{ Tactor } & \multicolumn{3}{|c|}{ Tactor } & \multicolumn{3}{|c|}{ Tactor } & \multicolumn{3}{|c|}{ Tactor } \\
\hline & & $0 \mathrm{~Hz}$ & $260 \mathrm{~Hz}$ & $280 \mathrm{~Hz}$ & $0 \mathrm{~Hz}$ & $260 \mathrm{~Hz}$ & $280 \mathrm{~Hz}$ & $\mathrm{OHz}$ & $260 \mathrm{~Hz}$ & $280 \mathrm{~Hz}$ & $0 \mathrm{~Hz}$ & $260 \mathrm{~Hz}$ & $280 \mathrm{~Hz}$ \\
\hline 95_EllipticalArea & Mean & 1.056 & 1.042 & 2.167 & 17.21 & 13.27 & 75.54 & 1.091 & 1.407 & 23.32 & 2.608 & 2.988 & 15.00 \\
\hline$m m^{2}$ & Std Dev & 0.520 & 0.582 & 2.205 & 17.78 & 20.98 & 214.7 & 0.583 & 0.889 & 65.11 & 2.654 & 2.623 & 39.24 \\
\hline 99_EllipticalArea & Mean & 1.623 & 1.602 & 3.331 & 26.45 & 20.40 & 116.1 & 1.677 & 2.162 & 35.84 & 4.009 & 4.594 & 23.05 \\
\hline$m m^{2}$ & Std Dev & 0.799 & 0.895 & 3.390 & 27.34 & 32.26 & 330.0 & 0.897 & 1.367 & 100.1 & 4.079 & 4.032 & 60.33 \\
\hline RMS_COP & Mean & 0.030 & 0.025 & 0.032 & 0.038 & 0.028 & 0.029 & 0.027 & 0.027 & 0.032 & 0.038 & 0.033 & 0.025 \\
\hline $\mathrm{mm}$ & Std Dev & 0.029 & 0.021 & 0.024 & 0.039 & 0.022 & 0.017 & 0.017 & 0.022 & 0.023 & 0.040 & 0.031 & 0.015 \\
\hline RMS_COP_ML & Mean & 0.011 & 0.008 & 0.010 & 0.010 & 0.013 & 0.011 & 0.007 & 0.009 & 0.012 & 0.014 & 0.012 & 0.010 \\
\hline $\mathrm{mm}$ & Std Dev & 0.005 & 0.004 & 0.007 & 0.009 & 0.011 & 0.006 & 0.004 & 0.006 & 0.008 & 0.014 & 0.006 & 0.007 \\
\hline RMS_COP_AP & Mean & 0.026 & 0.022 & 0.030 & 0.035 & 0.024 & 0.025 & 0.026 & 0.025 & 0.028 & 0.032 & 0.029 & 0.021 \\
\hline $\mathrm{mm}$ & Std Dev & 0.030 & 0.022 & 0.024 & 0.039 & 0.021 & 0.020 & 0.018 & 0.022 & 0.024 & 0.040 & 0.033 & 0.017 \\
\hline Sway_Velocity & Mean & 0.003 & 0.003 & 0.004 & 0.011 & 0.009 & 0.008 & 0.003 & 0.003 & 0.006 & 0.004 & 0.004 & 0.005 \\
\hline $\mathrm{mm} / \mathrm{s}$ & Std Dev & 0.002 & 0.002 & 0.002 & 0.008 & 0.007 & 0.008 & 0.002 & 0.002 & 0.008 & 0.002 & 0.002 & 0.004 \\
\hline Sway_Path_Length & Mean & 0.122 & 0.130 & 0.148 & 0.452 & 0.365 & 0.321 & 0.130 & 0.137 & 0.227 & 0.180 & 0.180 & 0.205 \\
\hline $\mathrm{mm}$ & Std Dev & 0.520 & 0.582 & 2.205 & 17.78 & 20.98 & 214.7 & 0.583 & 0.889 & 65.11 & 2.654 & 2.623 & 39.24 \\
\hline Circular_Area & Mean & 0.122 & 0.130 & 0.148 & 0.452 & 0.365 & 0.321 & 0.130 & 0.137 & 0.227 & 0.180 & 0.180 & 0.205 \\
\hline$m m^{2}$ & Std Dev & 0.061 & 0.061 & 0.079 & 0.303 & 0.293 & 0.311 & 0.064 & 0.067 & 0.301 & 0.090 & 0.076 & 0.167 \\
\hline DominantFreq_COP_R & Mean & 0.175 & 0.102 & 0.194 & 0.166 & 0.102 & 0.111 & 0.157 & 0.083 & 0.102 & 0.120 & 0.104 & 0.166 \\
\hline$H z$ & Std Dev & 0.277 & 0.037 & 0.272 & 0.220 & 0.037 & 0.042 & 0.221 & 0.000 & 0.037 & 0.060 & 0.059 & 0.190 \\
\hline DominantFreq_COP_AP & Mean & 0.083 & 0.092 & 0.102 & 0.092 & 0.231 & 0.111 & 0.166 & 0.083 & 0.083 & 0.111 & 0.083 & 0.102 \\
\hline$H z$ & Std Dev & 0.000 & 0.028 & 0.037 & 0.028 & 0.352 & 0.042 & 0.220 & 0.000 & 0.000 & 0.059 & 0.000 & 0.037 \\
\hline DominantFreq_COP_MI & Mean & 0.212 & 0.138 & 0.286 & 0.166 & 0.203 & 0.111 & 0.157 & 0.194 & 0.277 & 0.314 & 0.114 & 0.166 \\
\hline$H z$ & Std Dev & 0.266 & 0.072 & 0.322 & 0.220 & 0.269 & 0.042 & 0.192 & 0.212 & 0.345 & 0.387 & 0.043 & 0.190 \\
\hline MeanFreq_COP_R & Mean & 0.450 & 0.519 & 0.377 & 0.404 & 0.427 & 0.404 & 0.406 & 0.436 & 0.364 & 0.429 & 0.434 & 0.396 \\
\hline$H z$ & Std Dev & 0.223 & 0.299 & 0.218 & 0.211 & 0.186 & 0.218 & 0.237 & 0.155 & 0.138 & 0.202 & 0.195 & 0.147 \\
\hline MeanFreq_COP_AP & Mean & 0.309 & 0.391 & 0.315 & 0.341 & 0.361 & 0.307 & 0.411 & 0.383 & 0.332 & 0.362 & 0.323 & 0.330 \\
\hline$H z$ & Std Dev & 0.122 & 0.243 & 0.131 & 0.128 & 0.179 & 0.181 & 0.243 & 0.156 & 0.129 & 0.158 & 0.143 & 0.099 \\
\hline MeanFreq_COP_ML & Mean & 0.690 & 0.637 & 0.669 & 0.646 & 0.557 & 0.488 & 0.585 & 0.651 & 0.571 & 0.593 & 0.564 & 0.505 \\
\hline$H z$ & Std Dev & 0.243 & 0.205 & 0.272 & 0.217 & 0.184 & 0.196 & 0.195 & 0.208 & 0.212 & 0.169 & 0.136 & 0.156 \\
\hline
\end{tabular}

Table 3.2: Mini Logger Results from MANOVA 


\begin{tabular}{|c|c|c|c|c|c|c|c|c|c|c|c|c|c|}
\hline \multicolumn{14}{|c|}{ Xsens } \\
\hline \multicolumn{14}{|c|}{ Eyes Condition } \\
\hline & & \multicolumn{6}{|c|}{ Eyes Closed } & \multicolumn{6}{|c|}{ Eyes Open } \\
\hline & & \multicolumn{6}{|c|}{ Somatosensory Condition } & \multicolumn{6}{|c|}{ Somatosensory Condition } \\
\hline & & \multicolumn{3}{|c|}{ Control } & \multicolumn{3}{|c|}{ StepRight } & \multicolumn{3}{|c|}{ Control } & \multicolumn{3}{|c|}{ StepRight } \\
\hline & & \multicolumn{3}{|c|}{ Tactor } & \multicolumn{3}{|c|}{ Tactor } & \multicolumn{3}{|c|}{ Tactor } & \multicolumn{3}{|c|}{ Tactor } \\
\hline & & $0 \mathrm{~Hz}$ & $260 \mathrm{~Hz}$ & $280 \mathrm{~Hz}$ & $0 \mathrm{~Hz}$ & $260 \mathrm{~Hz}$ & $280 \mathrm{~Hz}$ & $\mathrm{OHz}$ & $260 \mathrm{~Hz}$ & $280 \mathrm{~Hz}$ & $\mathrm{OHz}$ & $260 \mathrm{~Hz}$ & $280 \mathrm{~Hz}$ \\
\hline 95_EllipticalArea & Mean & 1.981 & 2.102 & 2.447 & 9.697 & 8.920 & 5.616 & 2.775 & 1.435 & 1.366 & 4.911 & 3.203 & 2.578 \\
\hline$m m^{2}$ & Std Dev & 1.184 & 1.646 & 2.674 & 6.285 & 6.712 & 3.103 & 2.563 & 0.713 & 0.655 & 6.136 & 1.684 & 1.23 \\
\hline 99_EllipticalArea & Mean & 3.046 & 3.231 & 3.761 & 14.91 & 13.71 & 8.634 & 4.265 & 2.206 & 2.101 & 7.549 & 4.923 & 3.963 \\
\hline$m m^{2}$ & Std Dev & 1.821 & 2.530 & 4.110 & 9.662 & 10.32 & 4.771 & 3.940 & 1.095 & 1.007 & 9.432 & 2.589 & 1.891 \\
\hline \multirow[t]{2}{*}{ RMS_COP (mm) } & Mean & 0.029 & 0.023 & 0.027 & 0.031 & 0.022 & 0.026 & 0.023 & 0.026 & 0.026 & 0.035 & 0.030 & 0.024 \\
\hline & Std Dev & 0.028 & 0.019 & 0.019 & 0.034 & 0.018 & 0.016 & 0.015 & 0.022 & 0.020 & 0.037 & 0.031 & 0.016 \\
\hline RMS_COP_ML & Mean & 0.007 & 0.007 & 0.009 & 0.010 & 0.008 & 0.009 & 0.006 & 0.007 & 0.010 & 0.010 & 0.009 & 0.008 \\
\hline $\mathrm{mm}$ & Std Dev & 0.004 & 0.004 & 0.005 & 0.006 & 0.005 & 0.006 & 0.004 & 0.004 & 0.005 & 0.009 & 0.005 & 0.005 \\
\hline RMS_COP_AP & Mean & 0.027 & 0.022 & 0.024 & 0.028 & 0.019 & 0.022 & 0.021 & 0.023 & 0.022 & 0.032 & 0.027 & 0.021 \\
\hline $\mathrm{mm}$ & Std Dev & 0.029 & 0.019 & 0.020 & 0.035 & 0.020 & 0.020 & 0.016 & 0.024 & 0.022 & 0.036 & 0.032 & 0.018 \\
\hline Sway_Velocity & Mean & 0.013 & 0.013 & 0.012 & 0.042 & 0.035 & 0.026 & 0.014 & 0.012 & 0.012 & 0.019 & 0.018 & 0.017 \\
\hline $\mathrm{mm} / \mathrm{s}$ & Std Dev & 0.007 & 0.008 & 0.007 & 0.029 & 0.020 & 0.012 & 0.008 & 0.006 & 0.006 & 0.010 & 0.008 & 0.009 \\
\hline Sway_Path_Length & Mean & 0.505 & 0.523 & 0.494 & 1.666 & 1.383 & 1.059 & 0.546 & 0.474 & 0.487 & 0.769 & 0.713 & 0.698 \\
\hline$m m^{2}$ & Std Dev & 0.279 & 0.326 & 0.273 & 1.153 & 0.786 & 0.481 & 0.330 & 0.248 & 0.229 & 0.395 & 0.327 & 0.350 \\
\hline Circular_Area & Mean & 0.505 & 0.523 & 0.494 & 1.666 & 1.383 & 1.059 & 0.546 & 0.474 & 0.487 & 0.769 & 0.713 & 0.698 \\
\hline$m m^{2}$ & Std Dev & 0.279 & 0.326 & 0.273 & 1.153 & 0.786 & 0.481 & 0.330 & 0.248 & 0.229 & 0.395 & 0.327 & 0.350 \\
\hline DominantFreq_COP_R & Mean & 0.185 & 0.083 & 0.175 & 0.701 & 0.175 & 0.203 & 0.157 & 0.157 & 0.129 & 0.120 & 0.102 & 0.129 \\
\hline$H z$ & Std Dev & 0.123 & 0.000 & 0.128 & 1.493 & 0.140 & 0.195 & 0.077 & 0.113 & 0.094 & 0.084 & 0.037 & 0.111 \\
\hline DominantFreq_COP_AP & Mean & 0.175 & 0.111 & 0.194 & 0.720 & 0.249 & 0.175 & 0.148 & 0.148 & 0.129 & 0.111 & 0.120 & 0.129 \\
\hline$H z$ & Std Dev & 0.128 & 0.083 & 0.125 & 1.487 & 0.176 & 0.128 & 0.081 & 0.091 & 0.094 & 0.083 & 0.060 & 0.111 \\
\hline DominantFreq_COP_MI & Mean & 0.655 & 0.544 & 0.138 & 0.314 & 0.748 & 0.175 & 0.120 & 0.102 & 0.129 & 0.286 & 0.092 & 0.157 \\
\hline$H z$ & Std Dev & 1.445 & 1.353 & 0.117 & 0.284 & 1.549 & 0.201 & 0.084 & 0.037 & 0.138 & 0.405 & 0.028 & 0.113 \\
\hline MeanFreq_COP_R & Mean & 1.090 & 1.074 & 1.092 & 1.753 & 1.779 & 1.633 & 1.145 & 1.170 & 1.357 & 1.179 & 1.213 & 1.425 \\
\hline$H z$ & Std Dev & 0.634 & 0.610 & 0.728 & 1.189 & 1.007 & 0.924 & 0.980 & 0.586 & 0.700 & 0.641 & 0.697 & 0.687 \\
\hline MeanFreq_COP_AP & Mean & 0.754 & 0.913 & 0.880 & 1.353 & 1.356 & 1.210 & 0.955 & 0.822 & 0.838 & 0.909 & 0.878 & 1.077 \\
\hline$H z$ & Std Dev & 0.398 & 0.632 & 0.784 & 1.096 & 0.891 & 0.851 & 0.974 & 0.631 & 0.471 & 0.533 & 0.495 & 0.612 \\
\hline MeanFreq_COP_ML & Mean & 2.408 & 2.272 & 1.950 & 2.395 & 2.090 & 2.185 & 2.132 & 2.223 & 2.380 & 1.997 & 1.887 & 2.024 \\
\hline$H z$ & Std Dev & 1.383 & 1.204 & 1.179 & 0.965 & 0.696 & 0.717 & 0.989 & 1.215 & 1.140 & 0.662 & 0.672 & 0.524 \\
\hline
\end{tabular}

Table 3.3: Xsens Results from MANOVA 
als with vibration frequency of $260 \mathrm{~Hz}$. These results suggest that there may be an ideal frequency at which stochastic resonance can be applied in order to promote good standing balance.

We also found higher postural sway velocity and path length for young healthy adults when standing on the StepRight with eyes closed. Whereas the use of the StepRight alone did not yield any significant changes in the postural control of healthy adults, the additional task of closing one's eyes was significantly challenging their balance during quiet stance, as demonstrated by marked increase in postural sway velocity and path length. As discussed, postural control and balance depends on the integration of sensory inputs from our visual, vestibular, and somatosensory systems (Shumway-Cook \& Woollacott, 2006). In the case of decreased access to somatosensory inputs provided by the StepRight, and the removal of visual input from eyes closed (EC) condition with limited sensory systems and modified weighting (or dependence) on vestibular and somatosensory inputs to maintain postural control. These changes in postural sway were sensitively identified by IMU. Wearable sensors are widely being used for investigating fall risk assessment and fall predictions (Liu et al., 2012, Ekvall Hansson et al., 2019). 


\section{CHAPTER 4}

\section{Validation of Sway using Motion Capture Camera System and Inertial Sensors}

\subsection{Introduction}

A synthetic benchmarks was used to identify the performance of the IMU for the use in quite standing. The sensor was mounted to the grail motion system where the results of the Xsense (Bellusci et al., 2018) is compared. Recurrence quantification analysis (RQA) was used to verify the similarity between the two signals. RQA is a method of identifying important features in a signal through phase space reconstruction. It is possible to identify features in a one dimensional time series by using time delayed copies as a stand-in dimension for unobserved values (Marwan et al., 2007).

RQA is a powerful method used in analysing recurring relationships in complex dynamic systems (Schultz et al., 2015). deterministic systems, regardless of the time scale can be predicted knowing the initial state. Even for chaotic systems with widely different outcomes, practically these can be evaluated for the short-term. 


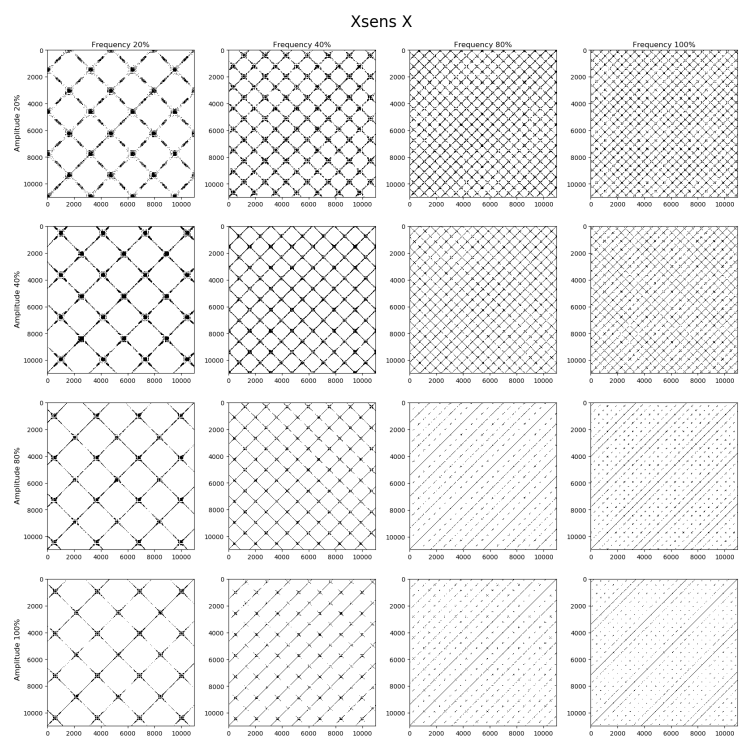

(a) RQA plots of inertial sensor sway signals

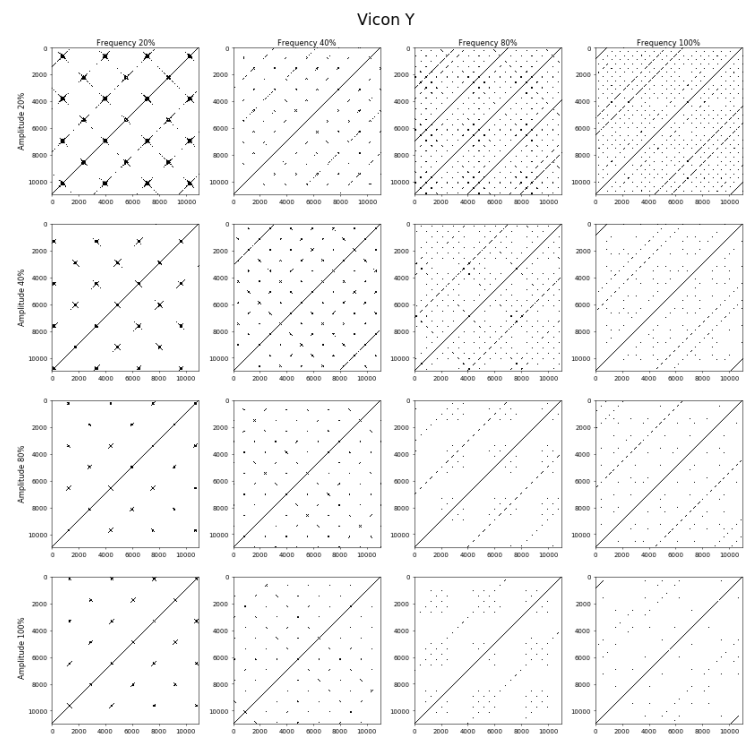

(b) RQA plots of marker sway signals

Figure 4.1: Recurrence Plot from synthetic motion

\subsubsection{RQA Analysis}

RQA was used to validate the similarity between the two signals. This can be achieved through analyzing the data into a higher dimension. This requires both finding the dimension to embed the signal in and amount of time to delay the embedding. Two methods are used to find these two methods; Average Mutual Information (AMI) and False Nearest Neighbor(FNN) function (Wallot \& Mønster, 2018, Marwan et al., 2007). RQA is used to quantify the duration and number of recurrences in the time series. A matrix with both frequency and amplitude between both Xsens and Vicon appear similar as seen in figure 4.1 and this can be verified with determinism where the profiles of both signals we're fairly similar as seen in figure 4.7.

\subsubsection{Average Mutual Information}

Average Mutual Information (AMI) is used to find optimal delay for embedding. Independence is quantified by $I(x(t), x(t+\tau))$ where $\tau$ is a constant that shifts the signal by some factor from original $x(t)$ and $x(t+\tau)$ (Wallot \& Mønster, 2018). This problem can be evaluated by a nonlinear generalization of the auto-correlation function and is given by equation 4.1 (Wallot \& Møn- 
ster, 2018).

$$
I(x(t), x(t+\tau))=\sum_{i, j}\left(p_{i j}(\tau) \log \left(\frac{p_{i j}(\tau)}{p_{i} p_{j}}\right)\right)
$$

\subsubsection{False Nearest Neighbors}

False Nearest Neighbors (FNN) is used to find the optimal embedding dimension of the signal (Wallot \& Mønster, 2018). This is reconstructed by embedding the original one-dimensional time series by taking time delayed signals (Wallot \& Mønster, 2018). This is described by the original time series $\mathrm{y}(\mathrm{t})$ and follows equation 4.2 .

$$
y(t)=(x(t), x(t+\tau), \ldots, x(t+(D-1) \tau))
$$

$\mathrm{t}$ and $\tau$ are integers that are indexed on the sample data (Wallot \& Mønster, 2018). The embedding dimension $\mathrm{D}$ can be estimated by examining the distance between points in phase space. If the embedding distance between the two neighbors changes appreciably then the neighbors are considered false neighbors and are true neighbors otherwise (Wallot \& Mønster, 2018). This is continued until the number of FNN drops to 0 (Wallot \& Mønster, 2018).

\subsubsection{Determinism}

$$
D E T=\frac{\sum_{l=l_{\text {min }}}^{N} l P(l)}{\sum_{l=1}^{N} l P(l)}
$$

$\mathrm{P}(\mathrm{l})$ is a measurement of diagonal lines segments 1 is the length of the line segment. $\mathrm{P}(1)$ is the number of occurrences of the given line segment. Processes with weak correlation tend to have shorter line segments (Marwan et al., 2007). Where processes with deterministic processes will tend to have long diagonal line segments with fewer isolated points (Marwan et al., 2007). The proportion of $l_{\min }$ points to all $\mathrm{p}(1)$ recurrence points is a measure of the predictability of the system (Marwan et al., 2007). 


\subsubsection{Recurrence Rate}

$$
R R_{\tau}=\sum_{i=1}^{N-\tau}\left(R_{i, i+\tau}\right)
$$

The distance between diagonal lines in RP space is refereed to as the recurrence rate (RR). $P_{\tau}(l)$ is defined as the number of points sampled on the diagonal line segment (Marwan et al., 2007). The main diagonal for the RP plot is fixed across all the trials and is excluded when examining the RR. $R R_{\tau}$ is described by 4.4 where $\tau=0$ is the main diagonal and $\tau>0$ is above the main diagonal and $\tau<0$ is the below the diagonal (Marwan et al., 2007). This measure directly relates to the likely hood that an event will occur again given some delay $\tau$. This can be generalized as a higher-order auto-correlation function as it is decadent on $\tau$ (Marwan et al., 2007).

\subsection{Methods and Materials}

\subsubsection{Grail Motion System}

The grail motion system is a treadmill system used for patients with neurological problems, orthopedic patients, and elderly. The repetitive motion of the treadmill is ideal setup of verifying the xsens IMU for standing motion. The Xsens and Vicon Tracking marker were both mounted onto the handrail of the treadmill as seen in figure 4.3. The Tracking marker was mounted close

to the Xsens to ensure that the signals were comparable. The treadmill has 10 degrees of forward and backward motion and $5 \mathrm{~cm}$ of horizontal transnational motion. The generator for the rotational axis is percent_magnitude $* 10 * \sin ($ percent_frequency $*$ TIME) and the transitional axis is percent_magnitude *.05*sin(percent_frequency *TIME). percent_magnitude is a percentage that modifies the maximum range for both transnational and rotational axes. percentages for both percent_magnitude and percent_frequency: 100\%,80\%, 40\%, 20\%. This translates to $10 \pm, 8 \pm, 4 \pm$ and $2 \pm$ degrees of rotational motion and $5 \pm, 4 \pm, 2 \pm, 1 \pm$ centimeters of horizontal motion. It takes 6 seconds for a whole rotation with the default time and the ranges 


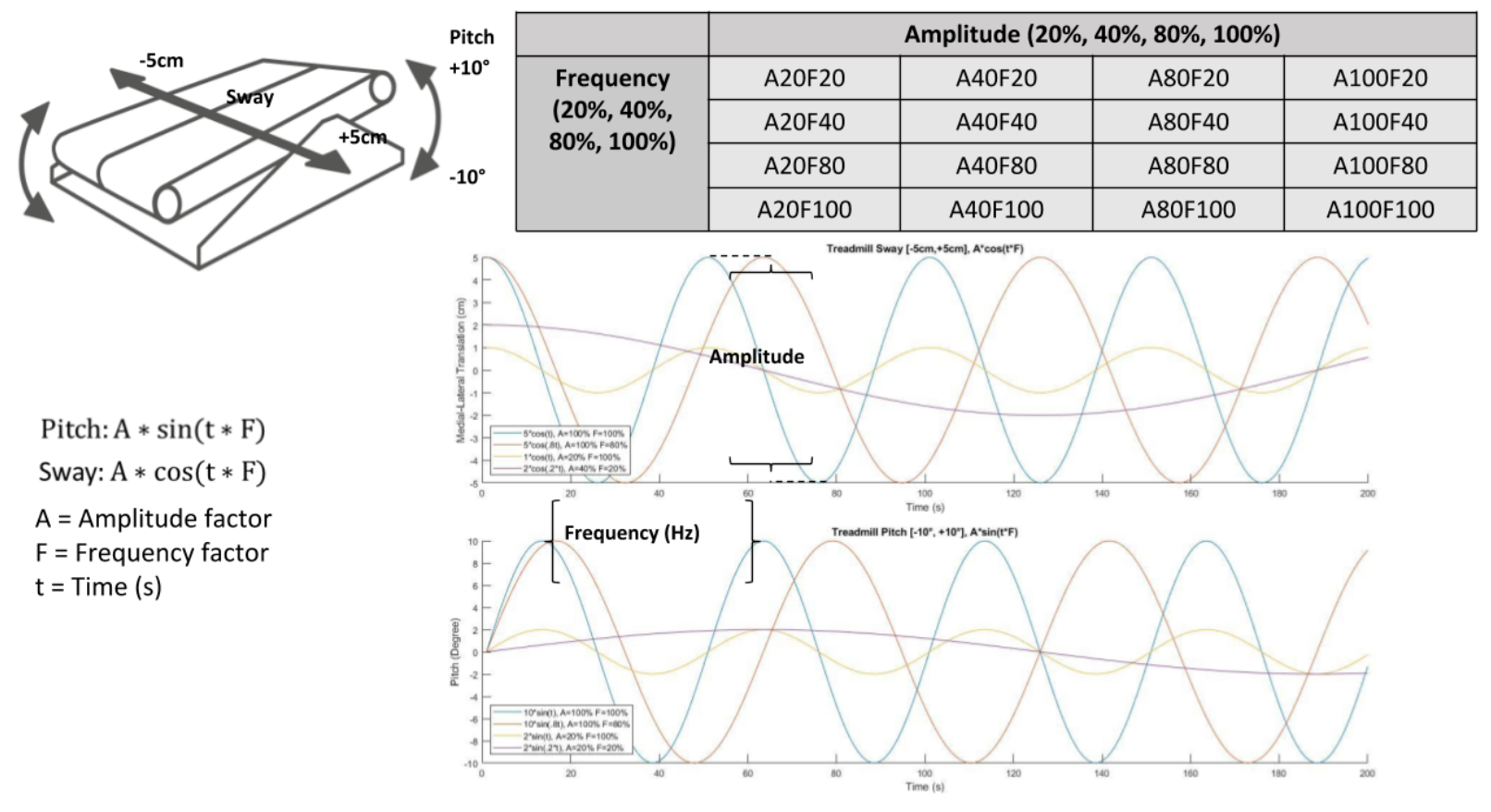

Figure 4.2: Sway Analysis Setup

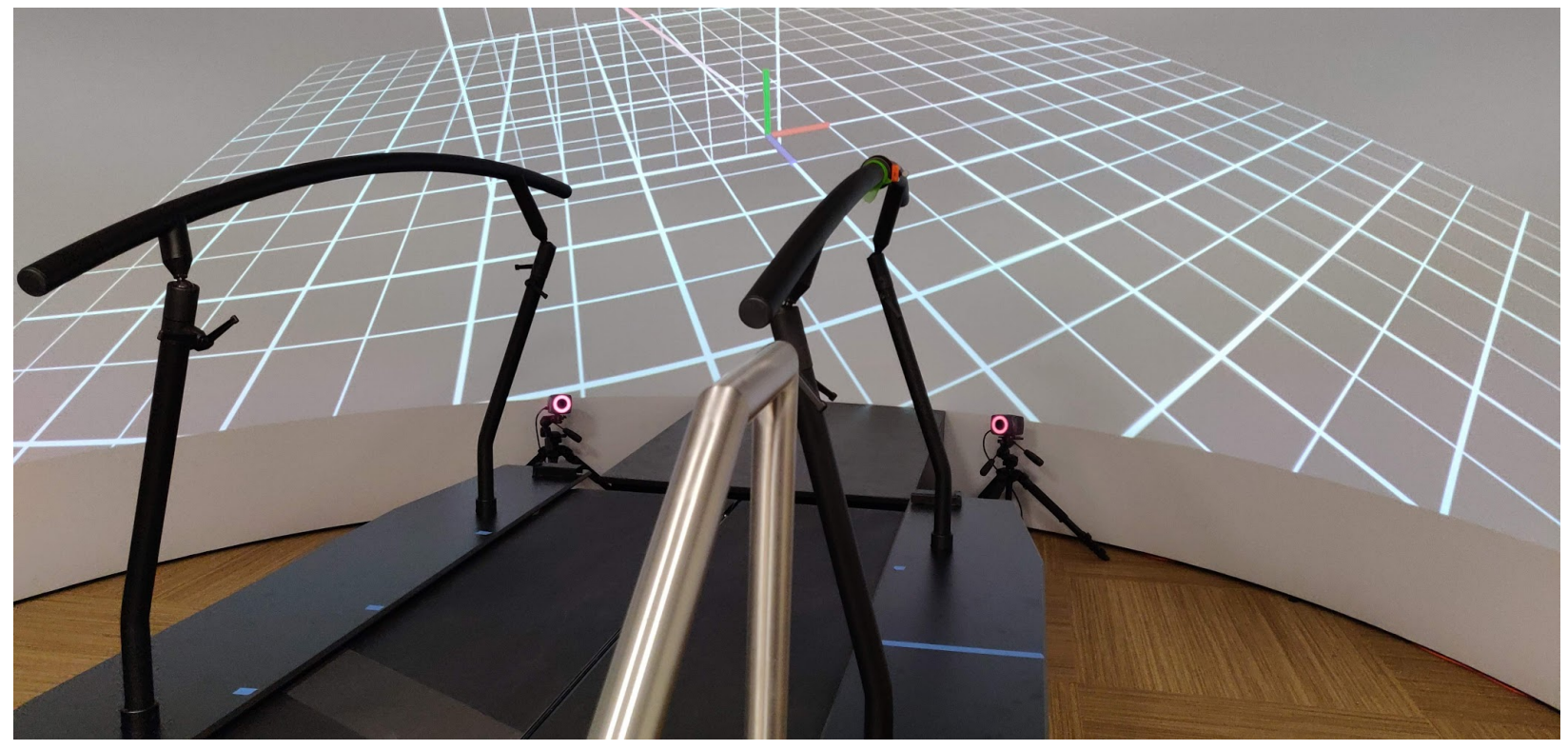

Figure 4.3: Treadmill Sensor Mounting 

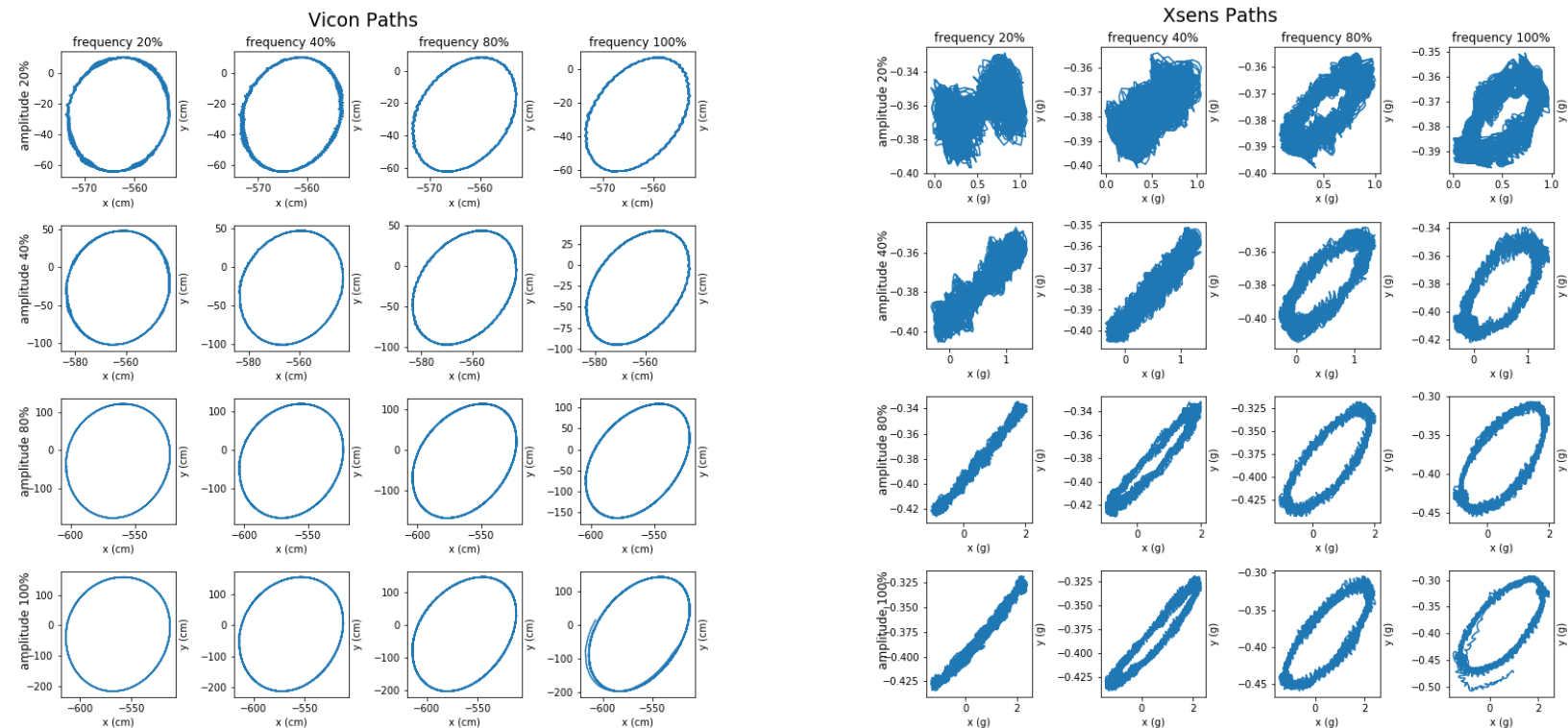

(a) filtered marker data
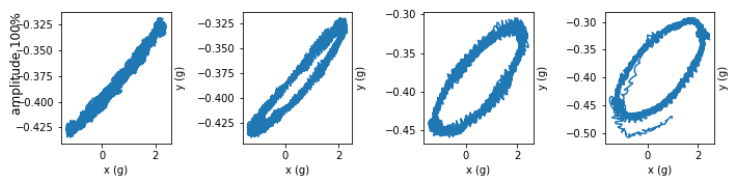

(b) filtered acceleration data

Figure 4.4: Filtered $x$ and $y$ data from Xsens and Vicon from synthetic motion

translates to $6,7.5,15$, and 30 seconds. The range of percentages allows the sensors to be validated against both the frequency and magnitude of motion.

The Xsens is mounted onto the handrail of the treadmill with a vicon tracking marking placed on top of the xsens senosor. The close proximity of

\subsection{Results}

\subsubsection{Signal Evaluation}

The filtered paths can be seen 4.4. The raw data is filtered with a 4th order low pass butterworth filter with a cutoff at 8 . the tracking marker used on the grail is a relatively fixed path so frequency does not play a strong factor. The relative path does not change with the frequency of the system where xsens is affected by this factor. The tracking marker is relative to a fixed frame of reference where the xsens is a local frame of reference local to the mounting of the sensor on the treadmill. Gravity plays a much larger factor that determines the rotational axis of the treadmill. The amplitude for the xsens plays a much larger factor in affecting both the rotational and transitional motion. the signal to noise ratio improves with a higher amplitude. 

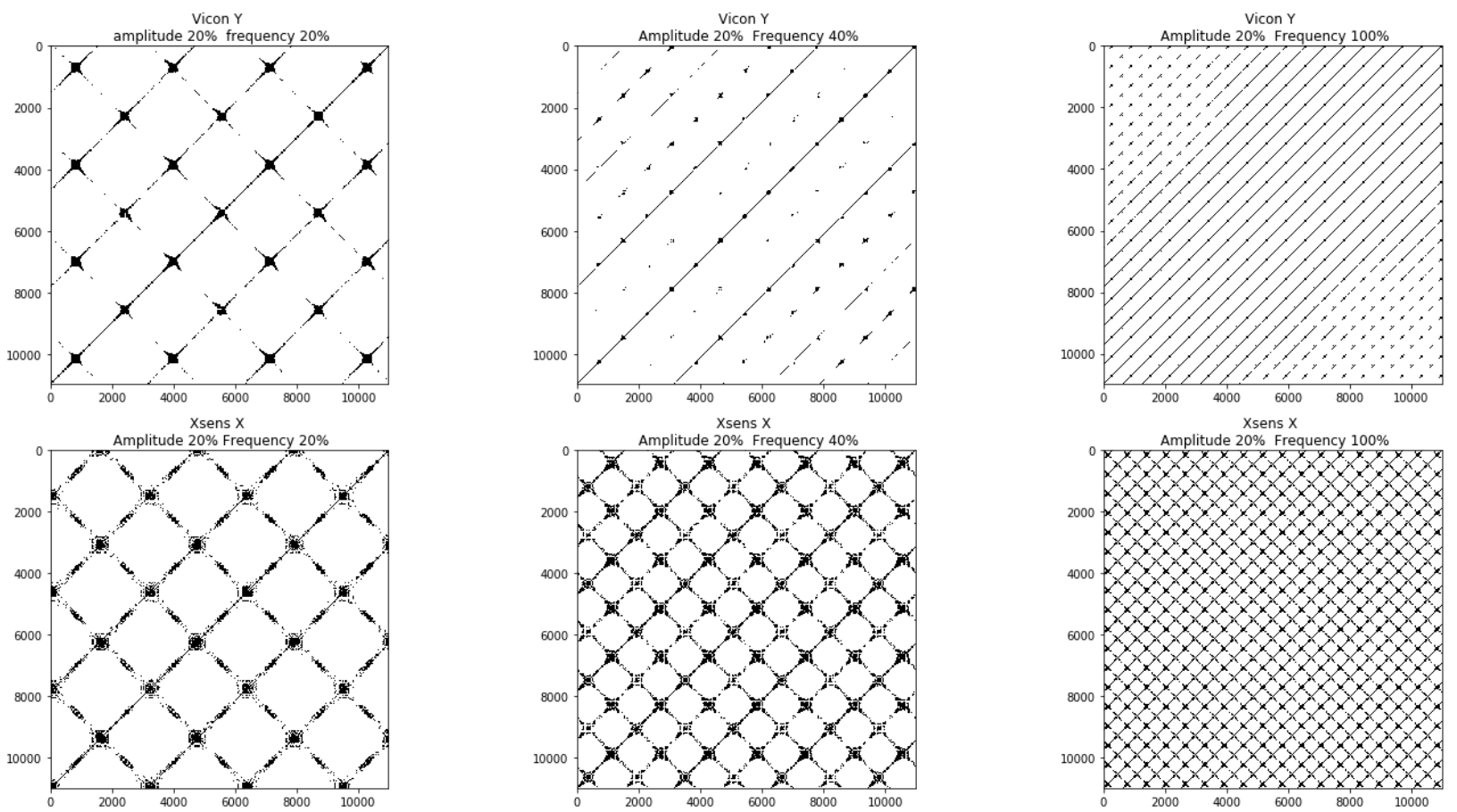

Figure 4.5: RQA subset from figure 4.1

\subsubsection{Recurrent plots}

The diagonal structure in the recurrence plots (RP) tend to suggest that the time series function has period to quasi-periodic recurrent structures (Marwan et al., 2007). This can be seen the with 4.5 where the distances between diagonal lines segments are fairly regular (Marwan et al., 2007). This regularity in the structure suggest that the signal source repeated at a fixed interval (Marwan et al., 2007). more quasi-period systems will have variations in the distance between diagonal segments (Marwan et al., 2007). The source for the signal was a fixed sin wave so this is expected when reviewing the RP data.

\subsubsection{Recurrence Rate}

The horizontal axis captures the sampled data and the vertical axis captures the rate of recurrence. A fixed signal was used in each trial so there is an expected period where the signal should repeat. The data from 4.6 was captured at a rate of $20 \%$ which translates to 15 second intervals from the first section. Xsens captures a lot of other residual noise where the peeks in the RP are 
Vicon $x$
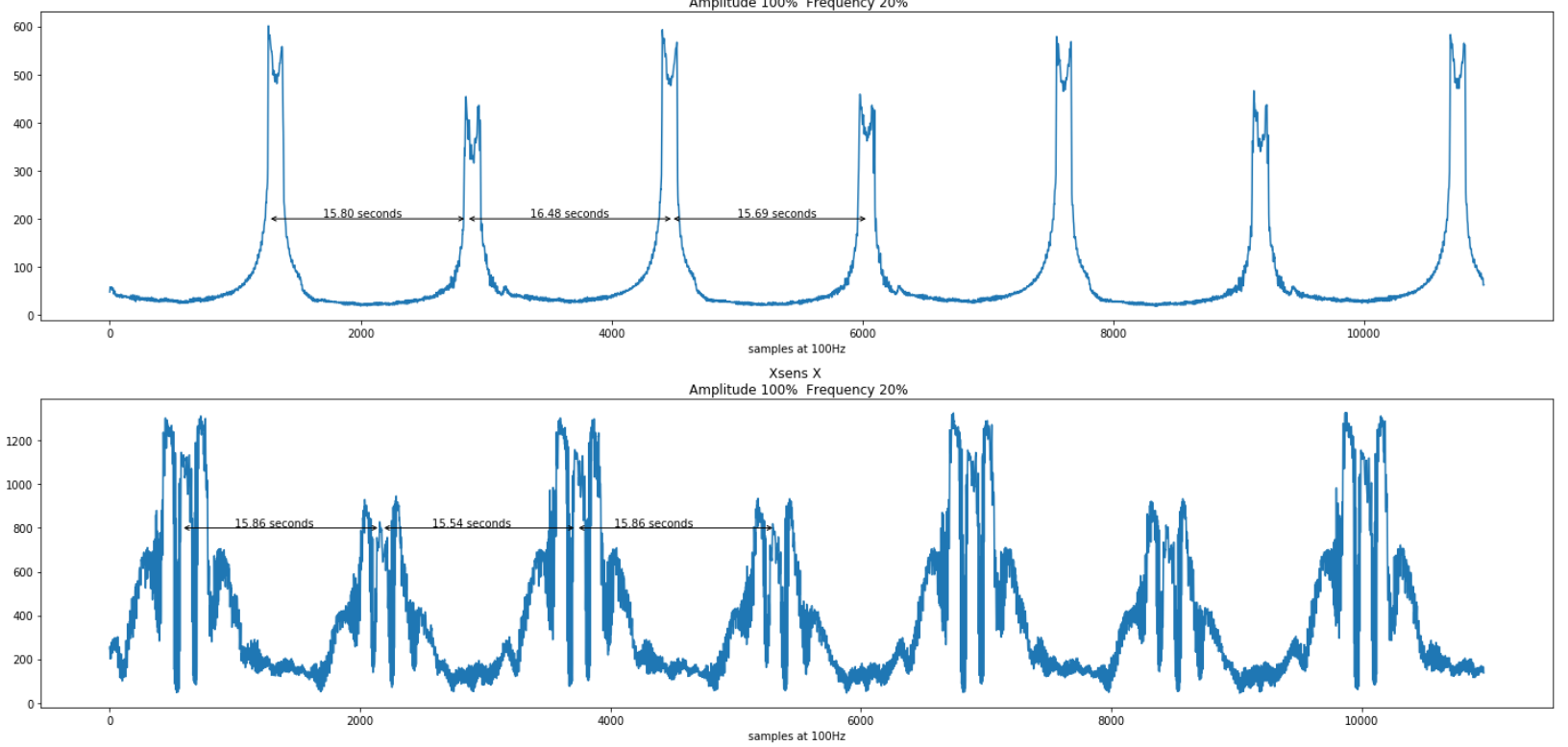

Figure 4.6: Recurrence rate

not as clean.

\subsubsection{Determinism}

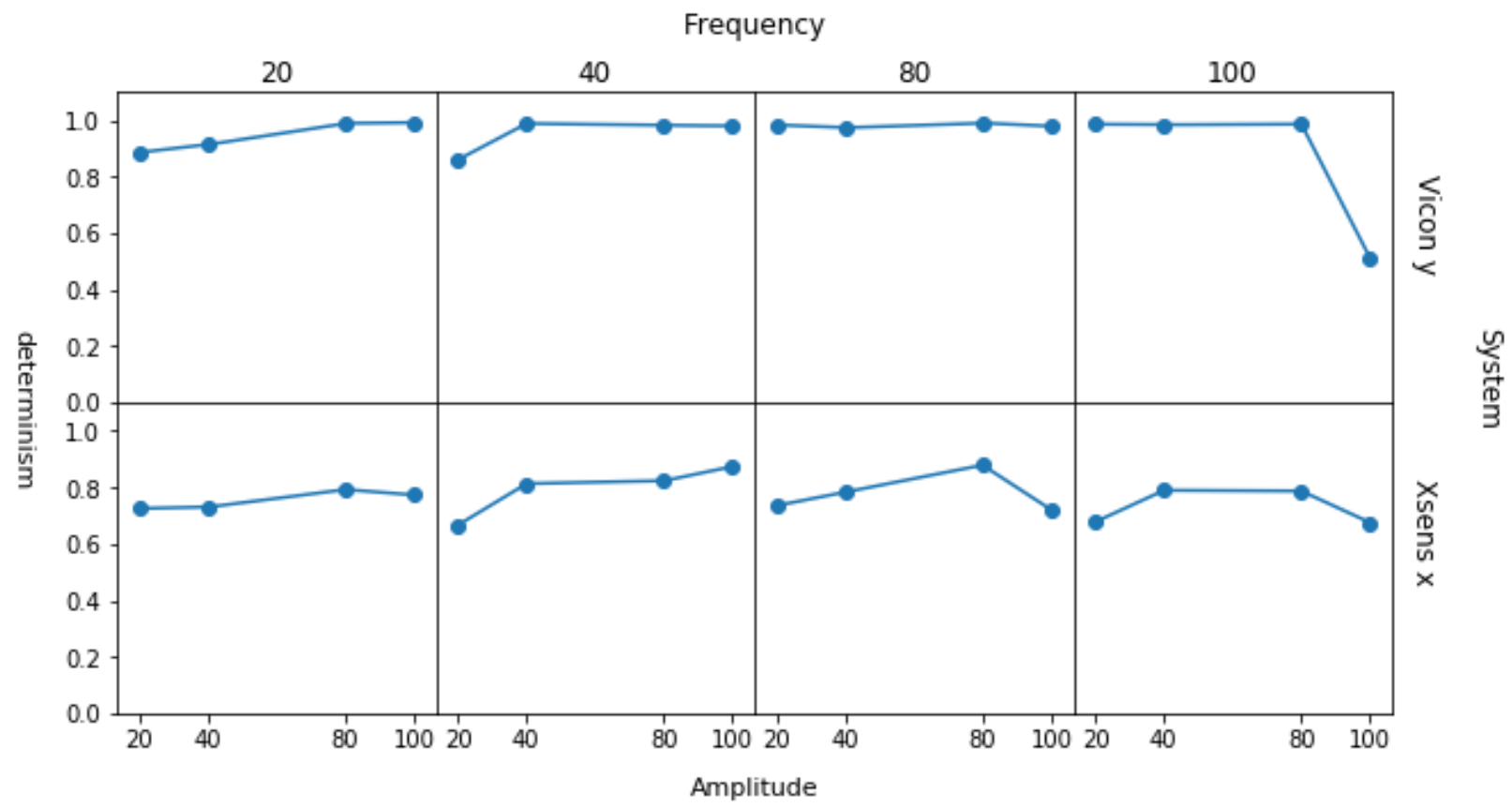

Figure 4.7: Determinism relation to frequency using a minimum diagonal length of 5 units. Vicon and Xsens shows similar profiles when combination of amplitude and frequency are combined, but xsens suffers more from signal noise. 
A value of 6 was chosen for $l_{\min }$ and the results can be seen with 4.7. xsens tends to have shorter segments because the signals tends to have more stochastic noise where the diagonal segments tend not to be as long. Determinism tends not to change a lot between ranges. Acceleration data is more sensitive to noise and this can be seen where determinism is below .8 where a tracking marker is closer to .9. This means that a large proportion of the segments are grater then $l_{\text {min }}$. 


\begin{tabular}{|c|c|c|c|c|}
\hline Amplitude & Frequency & Variable & Vicon Y & Xsens X \\
\hline \multirow[t]{8}{*}{20} & 20 & Determinism & 0.885527 & 0.725615 \\
\hline & & Recurrence Rate & 0.035679 & 0.026902 \\
\hline & 40 & Determinism & 0.859467 & 0.666460 \\
\hline & & Recurrence Rate & 0.016114 & 0.016657 \\
\hline & 80 & Determinism & 0.983201 & 0.735870 \\
\hline & & Recurrence Rate & 0.038141 & 0.032424 \\
\hline & 100 & Determinism & 0.985716 & 0.678274 \\
\hline & & Recurrence Rate & 0.033623 & 0.029954 \\
\hline \multirow[t]{8}{*}{40} & 20 & Determinism & 0.913222 & 0.730500 \\
\hline & & Recurrence Rate & 0.018916 & 0.018309 \\
\hline & 40 & Determinism & 0.988114 & 0.813703 \\
\hline & & Recurrence Rate & 0.021462 & 0.018803 \\
\hline & 80 & Determinism & 0.973281 & 0.784074 \\
\hline & & Recurrence Rate & 0.022922 & 0.015991 \\
\hline & 100 & Determinism & 0.983564 & 0.790227 \\
\hline & & Recurrence Rate & 0.015653 & 0.014428 \\
\hline \multirow[t]{8}{*}{80} & 20 & Determinism & 0.988763 & 0.793039 \\
\hline & & Recurrence Rate & 0.011061 & 0.009636 \\
\hline & 40 & Determinism & 0.981553 & 0.823598 \\
\hline & & Recurrence Rate & 0.013097 & 0.009109 \\
\hline & 80 & Determinism & 0.990064 & 0.879170 \\
\hline & & Recurrence Rate & 0.008971 & 0.007712 \\
\hline & 100 & Determinism & 0.986077 & 0.787656 \\
\hline & & Recurrence Rate & 0.010335 & 0.007327 \\
\hline \multirow[t]{8}{*}{100} & 20 & Determinism & 0.991442 & 0.773800 \\
\hline & & Recurrence Rate & 0.011006 & 0.007722 \\
\hline & 40 & Determinism & 0.979951 & 0.874375 \\
\hline & & Recurrence Rate & 0.010308 & 0.007359 \\
\hline & 80 & Determinism & 0.978718 & 0.721071 \\
\hline & & Recurrence Rate & 0.008687 & 0.005937 \\
\hline & 100 & Determinism & 0.512095 & 0.675977 \\
\hline & & Recurrence Rate & 0.000870 & 0.000399 \\
\hline
\end{tabular}

Table 4.1: result for determinism and recurrence rate 


\subsection{Discussion}

RQA was utilized to visualize the behavior of trajectories in n-dimensional phase space, which could represent dynamics of the moving platform. The RQA provides measures based on diagonal structures (Webber \& Zbilut, 1994). A signal is deterministic if there is no uncertainty with respect to its value at any instant of time. Recurrence plots can be generated which show for each moment in time, the times at which a phase space trajectory visits roughly the same area in the phase space (Fig 4.5). We found that recurrence plots are sensitive to the change of signal properties in the course of time, and are affected by high frequency noise. We performed RQA of the movement signals for 4 different frequencies and 4 different amplitude combinations of sway. We found that recurrence rate was highly dependent on noise and was affected by the noise in Mini-Logger, but determinism was found to be same for both the systems (Table 4.1 ). This study validated the measured signals originated from a common source.

\subsection{Conclusion}

The data was collected from a system with a cyclic pattern of motion. The xsens is collected from a local frame of reference where acceleration is heavily affected by noise and vicon is from a global frame a referenced from a tracked point. The data was validated from both xsens and Vicon to verify the relationship between the motion and the method of data collection. RQA was able to capture the cyclic nature of the signal and the clear diagonal lines in the RQA plots show that there is a strong recurrent nature to the data. The equal spacing between lines suggest that the signal is very predictable. 


\section{CHAPTER 5}

\section{Conclusion}

The platform that was built around the sensor has several limitations. The process of pressing a tactile button introduce noise into the data and extracting the SD card and saving the data introduce extra human error. Future work will correct some of these flaws in the protocol. The protocol and process for developing such a sensor is provided as a good starting point and should inform future work that attempts to improve on this model. There is a lot of space for improvement along with different methods that should produce a better outcome. Future work will examine other configurations such as a more compact design and wireless communcation would better appropriate the IMU.

There are other features for the ICM-20948 that can be tweaked to improve performance characteristics such as using the low band pass filter that comes with the IMU given that the the bandpass filter on the IMU can sample from a much higher sample rate then after the fact. Generally the performance can be improved by augmenting the data with other signals such as a pressure sensor, compass, or Magnetometer. the IMU can be paired with AK09916(magnetometer) and this should in theory also improve performance of the IMU. Atmega328 only has $32 \mathrm{~kb}$ of space and a fairly low end $8 \mathrm{Mhz}$ processor. the process of reading the writing the data to the SD card 
was pushing the limits of the Atmega processor and a better alternative would be needed.

Relying on just the acceleromter for the orientation of the sensor is not accurate in some cases. The starting orientation of the IMU can heavily skew the data and correcting the orientation with the average tilt is only a rough approximation. This method would be a lot better if this accounted for change in rotation from the gyroscope to correct for the change in tilt for an improvement in postural sway assessment.

A small trial with subjects can verify the validity of the sensor along with identifying problems with the setup and inform future improvements. an iterative step is necessary to work out the validity of the setup and verify feasibility of the setup. Our works concludes that wearable sensors developed in laboratories can be a cheap option for determination of postural sway and for the detailed fall risk in fall prone older population. 


\section{Appendix}

\section{Schematics \& Hardware}

\begin{tabular}{|l|l|l|}
\multicolumn{1}{|l}{ Designator } & MPN & 1 \\
\hline U2 & TLV70218DBVT & 1 \\
\hline U4 & ICM-20948 & 1 \\
\hline D3 & APT1608VBC/D & 1 \\
\hline Y1 & CSTNE8M00G550000R0 & 1 \\
\hline U3 & ATMEGA328-AU & 1 \\
\hline U1 & MIC5205-3.3YM5-TR & 1 \\
\hline SW2 & B3U-1000P & 1 \\
\hline J5 & DM3AT-SF-PEJM5 & 1 \\
\hline D1 & APT1608SGC & 1 \\
\hline U5 & PCA9306DCUR & 1 \\
\hline
\end{tabular}




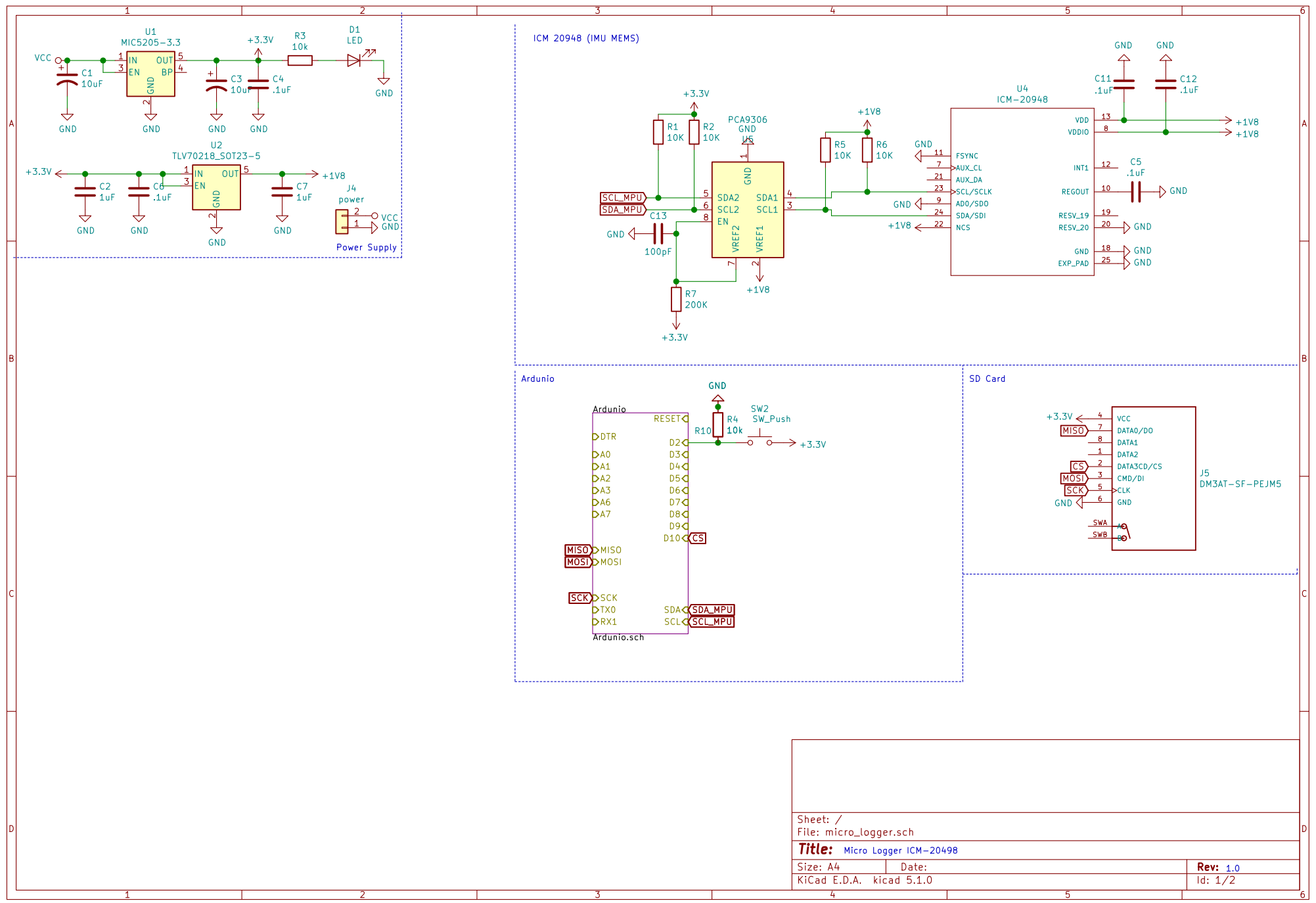




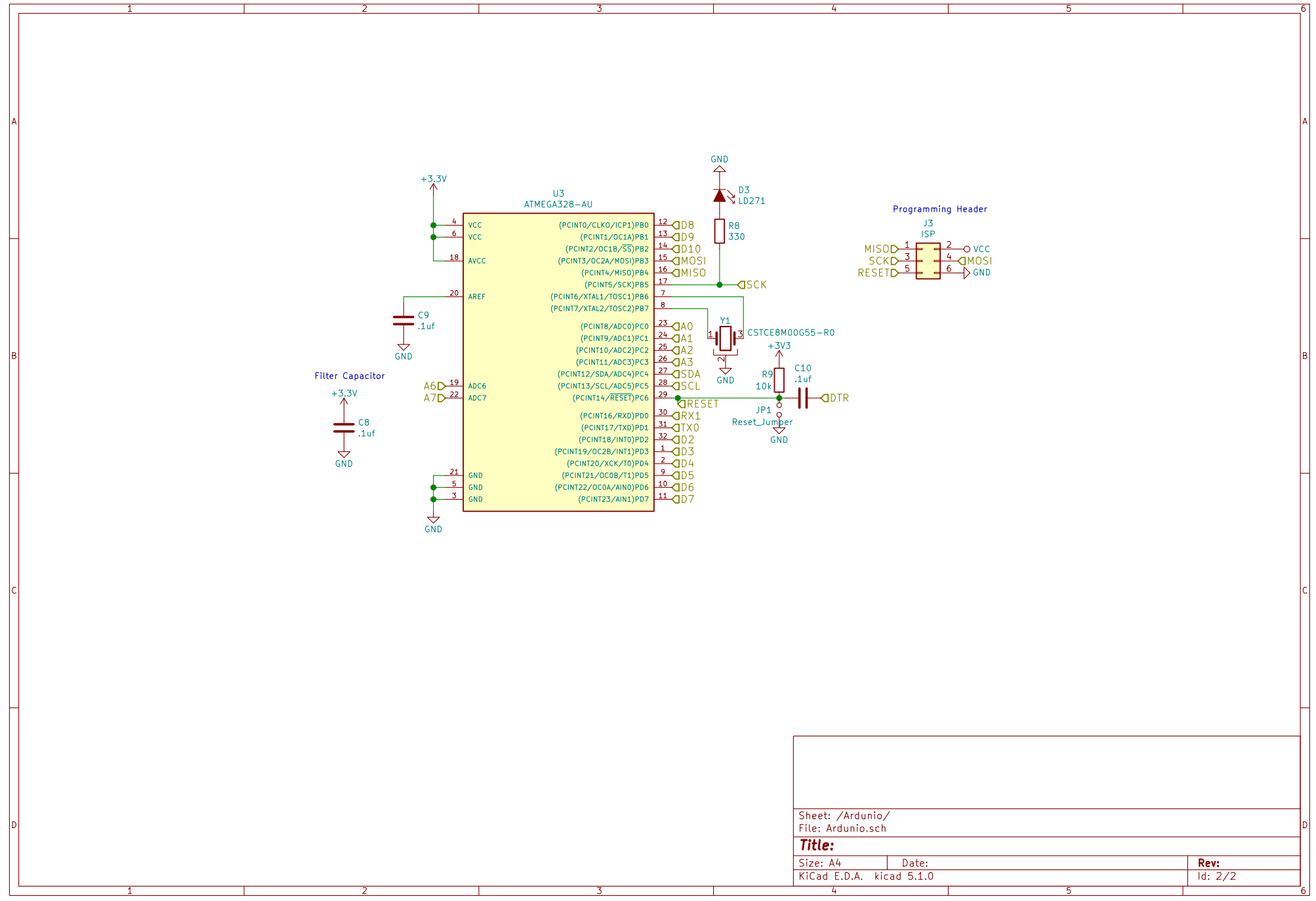




\section{REFERENCES}

(2004). Dual Bidirectional i2c Bus. Microchip.

(2010). Embedded 8bit RISC processor. Microchip.

(2010). Low Voltage Dropout Regulator. Texas Instruments.

(2014). i2c bus specification. NXP Semiconductor.

(2017). 9-Axis MEMS MotionTracking Device. TDK Sense.

(2018). SD Specification. Texas Instruments.

Bagherzadeh Cham, M., Mohseni-Bandpei, M. A., Bahramizadeh, M., Kalbasi, S., \& Biglarian, A. (2016). The clinical and biomechanical effects of subthreshold random noise on the plantar surface of the foot in diabetic patients and elder people: A systematic review. Prosthetics and Orthotics International, 40(6), 658-667.

Bellusci, G., Dijkstra, F., \& Slycke, P. (2018). Xsens MTw : Miniature Wireless Inertial Motion Tracker for Highly Accurate 3D Kinematic Applications. Xsens Technologies, (April), 1-9.

Cloutier, R., Horr, S., Niemi, J. B., Andrea, S. D., Lima, C., Harry, J. D., \& Veves, A. (2009). Diabetic Neuropathic Patients. The International Journal of Lower Extremity Wounds, 8(1), $6-10$.

Costa, M., Priplata, A. A., Lipsitz, L. A., Wu, Z., Huang, N. E., Goldberger, A. L., \& Peng, C. K. (2007). Noise and poise: Enhancement of postural complexity. EPL (Europhysics Letters), 77, 68008 .

Ekvall Hansson, E., Tornberg, A., \& Tornberg, $\square$. (2019). Coherence and reliability of a wearable inertial measurement unit for measuring postural sway. BMC Research Notes, 12(1), 201.

Grace Gaerlan, M., Alpert, P. T., Cross, C., Louis, M., \& Kowalski, S. (2012). Postural balance in young adults: The role of visual, vestibular and somatosensory systems. Journal of the American Academy of Nurse Practitioners, 24(6), 375-381. 
Guerraz, M. \& Bronstein, A. M. (2008). Ocular versus extraocular control of posture and equilibrium.

Lee, B. C., Martin, B. J., \& Sienko, K. H. (2013). The effects of actuator selection on nonvolitional postural responses to torso-based vibrotactile stimulation. Journal of NeuroEngineering and Rehabilitation, 10(1), 21.

Liu, J., Zhang, X., \& Lockhart, T. E. (2012). Fall Risk Assessments Based on Postural and Dynamic Stability Using Inertial Measurement Unit. Safety and Health at Work, 3(3), 192-198.

Martínez, L., Pérez, T., Mirasso, C. R., \& Manjarrez, E. (2007). Stochastic Resonance in the Motor System: Effects of Noise on the Monosynaptic Reflex Pathway of the Cat Spinal Cord. Journal of Neurophysiology, 97(6), 4007-4016.

Marwan, N., Carmen Romano, M., Thiel, M., \& Kurths, J. (2007). Recurrence plots for the analysis of complex systems. Physics Reports, 438(5-6), 237-329.

Mayagoitia, R. E., Lötters, J. C., Veltink, P. H., \& Hermens, H. (2002). Standing balance evaluation using a triaxial accelerometer. Gait and Posture, 16(1), 55-59.

Moe-Nilssen, R. (1998a). A new method for evaluating motor control in gait under real-life environmental conditions. Part 2: Gait analysis. Clinical Biomechanics, 13(4-5), 328-335.

Moe-Nilssen, R. (1998b). Test-retest reliability of trunk accelerometry during standing and walking. Archives of Physical Medicine and Rehabilitation, 79(11), 1377-1385.

Oskoei, M. A. \& Hu, H. (2008). Support vector machine-based classification scheme for myoelectric control applied to upper limb. IEEE Transactions on Biomedical Engineering, 55(8), 1956-1965.

Peterka, R. J. (2002). Sensorimotor Integration in Human Postural Control. Journal of Neurophysiology, 88(3), 1097-1118.

Phinyomark, A., Quaine, F., Charbonnier, S., Serviere, C., Tarpin-Bernard, F., \& Laurillau, Y. (2013). EMG feature evaluation for improving myoelectric pattern recognition robustness. Expert Systems with Applications, 40(12), 4832-4840.

Postema, K., Hijmans, J., Zijlstra, W., Hof, A., \& Geertzen, J. (2009). The effects of vibrating insoles on standing balance in diabetic neuropathy. Gait \& Posture, 30(9), S38-S39.

Priplata, A. A., Patritti, B. L., Niemi, J. B., Hughes, R., Gravelle, D. C., Lipsitz, L. A., Veves, A., Stein, J., Bonato, P., \& Collins, J. J. (2006). Noise-enhanced balance control in patients with diabetes and patients with stroke. Annals of Neurology, 59(1), 4-12.

Rubenstein, L. Z. (2006). Falls in older people: Epidemiology, risk factors and strategies for prevention. In Age and Ageing, volume 35 (pp. ii37-ii41).: Narnia.

Schubert, P. \& Kirchner, M. (2014). Ellipse area calculations and their applicability in posturography. Gait and Posture, 39(1), 518-522. 
Schultz, D., Spiegel, S., Marwan, N., \& Albayrak, S. (2015). Approximation of diagonal line based measures in recurrence quantification analysis. Physics Letters, Section A: General, Atomic and Solid State Physics, 379(14-15), 997-1011.

Severini, G. \& Delahunt, E. (2018). Effect of noise stimulation below and above sensory threshold on postural sway during a mildly challenging balance task. Gait and Posture, 63, 27-32.

Shaffer, S. W. \& Harrison, A. L. (2007). Aging of the Somatosensory System: A Translational Perspective. Physical Therapy, 87(2), 193-207.

Shumway-Cook, A. \& Woollacott, M. (2006). Motor Control: Translating Research Into Clinical Practice, volume 18.

Wallot, S. \& Mønster, D. (2018). Calculation of Average Mutual Information (AMI) and FalseNearest Neighbors (FNN) for the Estimation of Embedding Parameters of Multidimensional Time Series in Matlab. Frontiers in Psychology, 9(SEP), 1679.

Webber, C. L. \& Zbilut, J. P. (1994). Dynamical assessment of physiological systems and states using recurrence plot strategies. Journal of Applied Physiology, 76(2), 965-973.

Wei, Q., Liu, D. H., Wang, K. H., Liu, Q., Abbod, M. F., Jiang, B. C., Chen, K. P., Wu, C., \& Shieh, J. S. (2012). Multivariate multiscale entropy applied to center of pressure signals analysis: An effect of vibration stimulation of shoes. Entropy, 14(11), 2157-2172.

Yao, H.-Y. \& Hayward, V. (2010). Design and analysis of a recoil-type vibrotactile transducer. The Journal of the Acoustical Society of America, 128(2), 619-627.

Zalewski, C. K. (2015). Aging of the Human Vestibular System. Seminars in Hearing, 36(3), 175-196. 